\title{
Surface Plasmons in metallic nanoparticles: Fundamentals and applications.
}

\author{
M. A. Garcia, \\ Dpt. Electroceramics, Institute for Ceramic and Glass, CSIC, C/Kelsen 5, 28049 Madrid, Spain \& \\ IMDEA Nanociencia, Madrid 28049, Spain \\ magarcia@icv.csic.es
}

Index

1. Introduction

2. Fundamentals of SP in nanoparticles

2.1 SP in nanoparticles

2.2 Models and calculations of SP.

2.3 Size effects

2.4 Shape effects

2.5 Media effects

2.6 NPs Interaction effects

3. Applications of SP

3.1 Biomedical: Diagnosis and treatment

3.2 Energy: Photo-energies

3.3 Environmental Photocatalysis

3.4 Information technologies

4. Outlook and challenges

5. Acknowledgments

6. References 


\begin{abstract}
The excitation of surface plasmons in metallic nanoparticles induces optical properties hardly achievable in other optical materials, yielding a wide range of applications in many fields. This review presents an overview of surface plasmons in metallic nanoparticles. The concept of surface plasmons in nanoparticles is qualitatively described using a comparison with simple linear oscillators. The mathematical models to carry on calculations on surface plasmons are presented as well as the most common approximations. The different parameters governing the features of surface plasmons and their effect on the optical properties of the materials are reviewed. Finally, applications of surface plasmons in different fields as biomedicine, energy, environment protection and information technology are revised.
\end{abstract}




\section{Introduction}

Surface Plasmons (SP) resonance is the most outstanding optical property of metallic nanostructures. It consists of a collective oscillation of conduction electrons excited by the electromagnetic field of light. SP resonance is in the origin of optical properties hardly achievable with other physical processes $[1,2,3]$. In the case of metallic nanoparticles (NPs), where the electrons are confined in the three dimensions, the electron oscillations induce an electric field around the NP that can be much larger than the incident light one. SP are one of the best examples that things are different at the nanoscale. When the size of a metallic particle is reduced to the few nanometre range, the optical properties are dramatically modified by the appearance of SP and its behaviour results completely different than the bulk metal one. SP open the possibility to amplify, concentrate and manipulate light at the nanoscale, overcoming the diffraction limit of traditional optics and increasing resolution and sensitivity of optical probes [4]. Consequently, the SP can be used in a wide range of fields, including biomedical $[5,6,7,8]$, energy $[9,10,11]$, environment protection $[12,13,14]$, sensing [15] and information technology $[3,4,16]$ applications. Nowadays, there are well established applications of SP that increases rapidly with the development of our capabilities to fabricate and manipulate nanomaterials.

In this review the fundamentals of SP are surveyed. It does not pretend to be a presentation of the proper and exact formalism required to describe SP but an attempt to explain what are SP in an accessible way to most readers with different background. To this purpose, analogies with simple systems as linear oscillators are used. After describing the physical basis of SP, the theories that provide exact mathematical solutions and the usual approximations to carry on proper calculations on SP are summarised. The main parameters affecting the SP excitation processes are also revised. Finally, some applications of SP on different fields are surveyed. It should be impossible to review here the huge number of applications of SP reported in the literature. Thus, when selecting just some of them I do not pretend to neglect the researchers in the field the credit they merit but just to highlight some applications to illustrate the capabilities of SP.

The synthesis of metallic NPs in not addressed here. There are excellent reviews of this topic that the reader is referred to $[17,18,19,20,21,22,23]$. Moreover, NPs are not the only metallic nanostructures exhibiting SP. Films, wires and patterned media exhibit also SP [2,24,25]. In the case of NPs, the confinement of electrons leads to localised SP, while other structures with large dimensions compared with light wavelength (wires and films) hold extended SP that propagate 
along the interface between the metal and the dielectric medium. There is a third case corresponding to mesoscopic systems, with dimensions comparable to wavelength. In this case SP modes can also be excited [2,3]. We will only treat here the case of localised SP in NPs.

\section{Fundamentals of SP in NPs}

The scientific study of SP started in early XX century when Gustav Mie published his pioneer work explaining the surprising optical properties of metallic colloids [26]. However, SP in NPs have been empirically used for a long time, particularly for glass colouring. Glasses from the final bronze age (1000-1200 BC) found in Fratessina di Rovigo (Northern Italy) were coloured by the presence of metallic Cu NPs [27]. The Lygurcus cup (400 AC) is probably the most famous example of the use of SP in the ancient times, exhibiting different coloration when observed upon illumination inside or outside of the cup $[28,29]$. Actually many Roman mosaics used metallic NPs to achieve red coloration $[30,31]$. At that time, Maya civilization also used metallic NPs to develop blue paints exploding SP [32]. There are also evidence of the ancient use of metallic NPs in glass and pottery from Egyptian dynasties [33], Celtic enamels [34], Japanese [35] and Chinese glasses [36].

In the Middle Ages, the development of glass manufacturing processes induced outstanding advances on stained glasses fabrication $[37,38]$. The variety of metallic NPs for glass colouring increased substantially as we can observe in many church windows from that period. The development of glass chemistry during Renaissance [39] and modern age [40], provided better tuning of coloration effects based on the SP of metallic NPs. Excellent revisions of the historical use of SP in metallic NPs for glass and pottery colouring are presented in ref. [31,41].

All these developments were empirically achieved without knowing the real origin of the surprising optical effects appearing when metallic atoms were introduced in the glass. After Mie's work in early XX century, the origin of the optical properties of metallic NP was understood, but further exploitation was limited by the capabilities to synthesise and manipulate NPs in a controlled way. It was not until the development of nanotechnology at the end of XX century that the applications of SP spread quickly on many fields.

\section{2. $1 \mathrm{SP}$ in NPs}

As above indicated, SP correspond to an interaction between matter and the electromagnetic field of the light. Thus, the exact analysis of SP implies solving the Maxwell equations with the appropriate boundary conditions [1]. The solution of these equations is only possible for certain conditions and even in this case, the results are mathematical series that do not explain what are 
SP. However, a simplified classical picture can be more useful to understand the physical meaning of SP. A metallic NP can be described as a lattice of ionic cores with conduction electron moving almost freely inside the NP (the Fermi sea) as figure 1 illustrates. When the particle is illuminated, the electromagnetic field of the light exerts a force on these conduction electrons moving them toward the NP surface. As these electrons are confined inside the NP, negative charge will be accumulated in one side and positive charge in the opposite one, creating an electric dipole. This dipole generates an electric field inside the NP opposite to that of the light that will force the electrons to return to the equilibrium position (figure 1). The larger the electron displacement, the larger the electric dipole and consequently the restoring force. The situation is similar to a linear oscillator with a restoring force proportional to the displacement from the equilibrium position. If the electrons are displaced from the equilibrium position and the field is later removed, they will oscillate with a certain frequency that is called the resonant frequency; in the case of SP it is named the plasmonic frequency. Actually, the electron movement inside the NP exhibits some damping degree. The ionic cores and the NP surface partially damp the electron oscillations. Thus, the system is similar to linear oscillator with some damping.
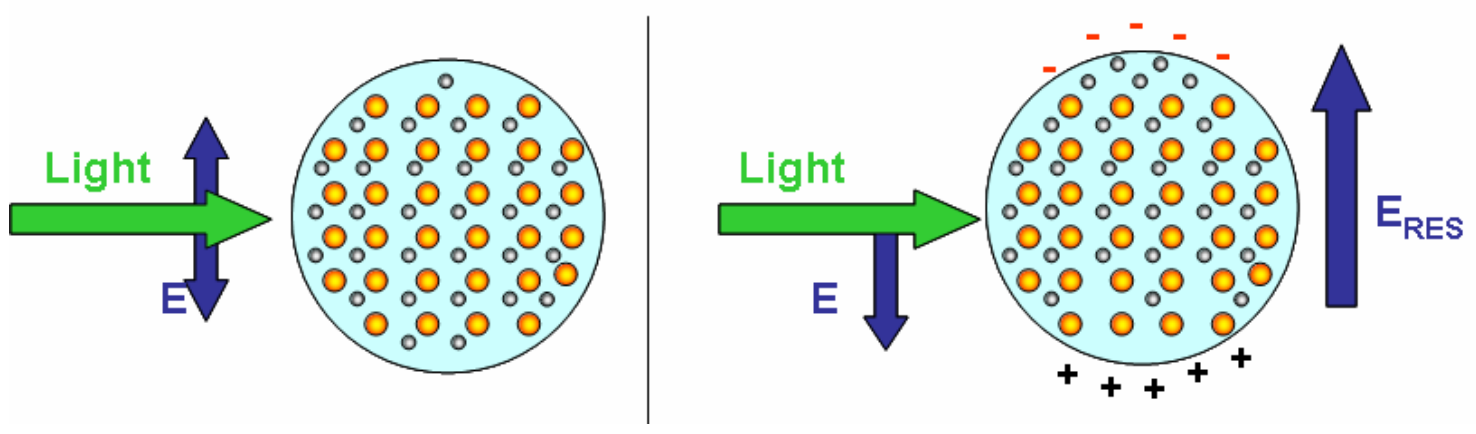

Figure 1. Scheme of the light interaction with a metallic NP. The electric field of the light induces the movement of conduction electrons which accumulate at the NP surface creating an electric dipole. This charge accumulation creates an electric field opposite to that of the light.

When an alternating force is applied to a linear oscillator, the system oscillates with the same frequency than the external force but the amplitude and phase will depend on both the force and the intrinsic parameters of the oscillator. In particular, the oscillating amplitude will be maxima for the resonant frequency (figure 2a). It is quite straightforward to understand that, if the frequency of the external force is the same that the plasmonic frequency of the NP, it will be easy to make the electrons oscillate, but as we move far way from this frequency the movement of electrons will be more difficult, i.e., with reduced amplitude. 
We can not observe directly the electrons movement to determine their oscillating amplitude. However, we can determine this amplitude indirectly. The electronic oscillation implies an increase of kinetic and electrostatic energies associated to the electric fields of the dipole. As energy must be conserved, this increase of energy must be provided by the illuminating light. Therefore, the light extinguishes partially when exciting SP inside the NP. The larger the electron oscillations, the larger the light extinction, so the optical absorption spectrum allows to detect the excitation of SP. The resonant frequency for these oscillations in metallic NPs corresponds typically to UV-Vis light and consequently, the SP arise absorption bands in this region of the spectrum as figure $2 b$ illustrates.
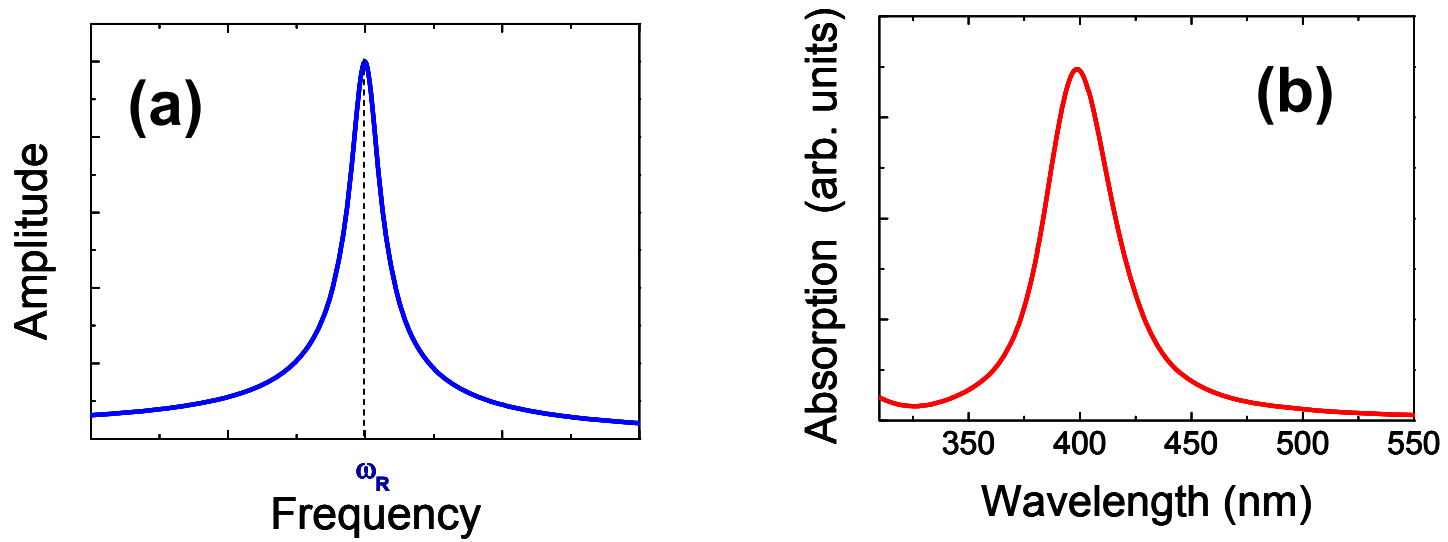

Figure 2. (a) Oscillation amplitude for a linear oscillator as a function of the external force frequency (b) Optical absorption spectrum corresponding to $10 \mathrm{~nm}$ silver NPs embedded in a silica glass.

At this stage, SP can be considered as another electronic process in which light is absorbed to promote electrons from the ground level to an excited one. What makes the SP unique are the numbers of these processes. The absorbing efficiency of a particle is given by its absorption cross section. Classically it corresponds to the geometrical section of an ideal opaque particle absorbing the same number of photons than the studied particle. As figure 3 illustrates, we could replace the absorbing NP by a perfect opaque one (absorbing any photon reaching its surface) that will absorb the same number of photons that our real particle. The section of this ideal particle represents the absorption cross section of the NP. For instance, if we have a NP absorbing half of the photons reaching its surface, the absorption cross section will be half of its geometrical section. Besides absorption, light interacting with matter can be scattered, changing the propagation direction and eventually also energy and moment. For this process, we can define also the scattering cross section as the geometrical section of an ideal scattering particle (that scatters any photon reaching its surface) with the same scattering efficiency than the real particle. 


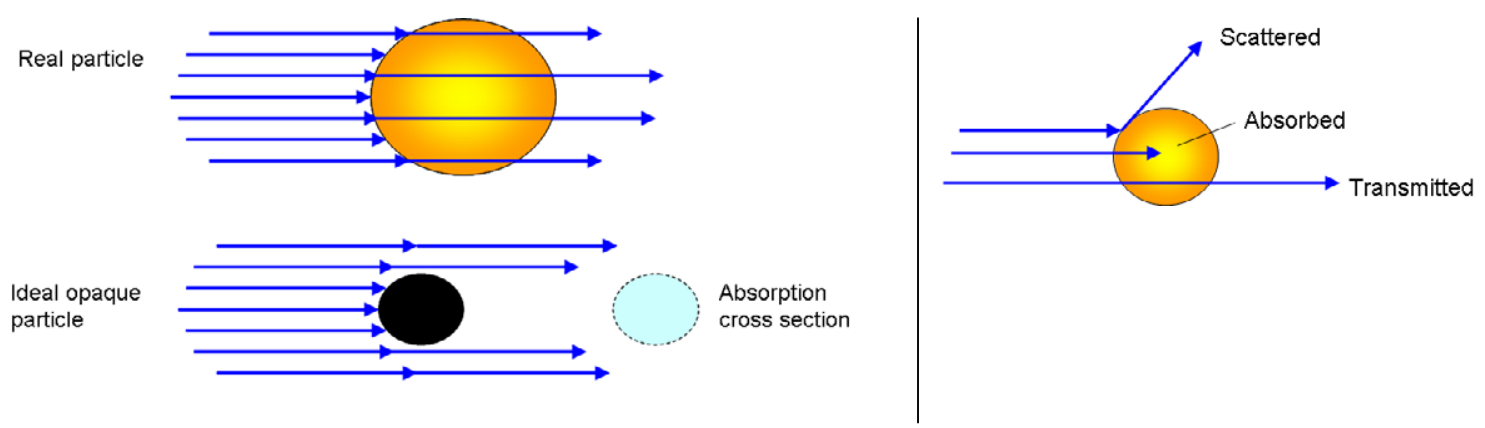

Figure 3. (left) Illustration of absorption cross section concept. (right) Picture describing transmission, absorption and scattering processes.

The sum of absorption and scattering cross section is defined as the extinction cross section that represents the efficiency of the particle to remove photons from an incident beam (by both, absorption or scattering processes). For perfect opaque particles the extinction cross section can be at maximum the particle section $\left(\pi \cdot R^{2}\right)$. However, for few nm NPs, extinction cross sections larger to $10 \%$ of the geometrical section are scarcely found for other processes different to SP excitation $[42,43]$.

Figure 4 present the resonant wavelength and the extinction cross section for $10 \mathrm{~nm}$ size metallic NPs. It is found that for noble metal NPs the extinction cross section can be up to 10 times their geometrical section; that is, the NP is capable of absorbing and scattering photon even away from its physical position. Somehow, the excitation of the SP is equivalent to concentrate the light passing by the NP to induce a huge extinction. It is worth noting that the light absorption has an exponential dependence on the absorption cross section. A light beam propagating across a medium with metallic NPs decay in intensity as:

$l(x)=I_{0} \cdot e^{-C \cdot \sigma \cdot x}$

being $I_{0}$ the initial intensity, $C$ the concentration of NPs per unit volume, $\sigma$ their extinction cross section and $x$ the travelled distance. Therefore, a moderate increase in the extinction cross section can lead to a huge enhancement of light absorption. As an example, if we have a dielectric matrix containing $10^{20} \mathrm{NPs}$ per $\mathrm{cm}^{3}$ with $10 \mathrm{~nm}$ size (1\% in volume) and their extinction cross section is equal to the geometrical section $\left(\pi \cdot R^{2}\right)$, the light transmitted across $1 \mu m$ of the material will be $45 \%$; if the extinction cross section of the NPs is increased a factor of 10 , the transmitted light is reduced to $0.04 \%$ (i.e., 3 orders of magnitude). These numbers make SP unique, since the cross section values found here are fairly larger than other optical processes as electronic transitions in semiconductors (band to band transitions), rare earths atoms, defect related absorption process in solid, or electronic excitations in molecules. 


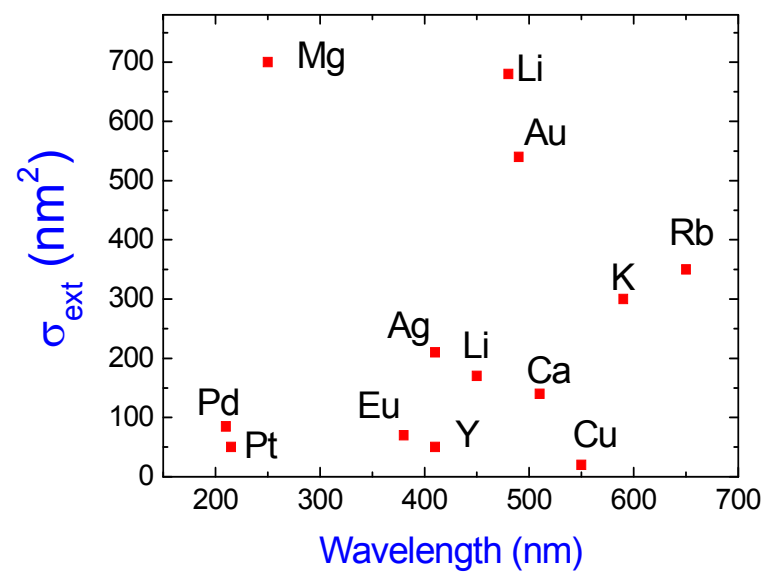

Figure 4. Resonant frequency and extinction cross section for metallic NPs with $10 \mathrm{~nm}$ size in air.

A more direct example of the huge extinction in metallic NPs due to SP excitation is provided by a comparison with interband transitions. Besides SP, there are other possible electronic excitations in metallic NPs. In a metallic material, valence and conduction bands overlap forming a continuous spectrum of available states. However, some inner levels do not split enough to overlap these bands so the system may exhibit interband transitions similar to those in semiconductors [44]. Transitions between these inner levels and the conduction band induce an absorption edge similarly to the case of semiconductors. Actually, some metals present a weak luminescence emission due to electron decay between these bands. For bulk materials these transitions are very unlikely and optical absorption and emission associated with these transitions result very weak. For instance, a weak photoluminescence associated to interband transitions has been measured for bulk gold [45] corresponding to transitions between the $3 d$ level and the conduction band. However, for NPs with a reduced number of atoms, energy bands are not so well formed because of the limited number of atoms and interband transitions become more prominent.

Figure 5 shows the optical absorption spectrum of $\mathrm{Ag}$ and Au NPs. In the case of Ag, both SP band and interband transition absorption edge are well resolved. For Au NPs, both absorptions overlap. This overlapping is important when analysing the shape of the SP. In particular, the width of the SP band (related to the NP size) must be determined separating the contribution of the interband transitions. We may observe how the SP absorption is fairly larger than that of the interband transitions despite the fact that the absorbing centre is the same for both processes. 

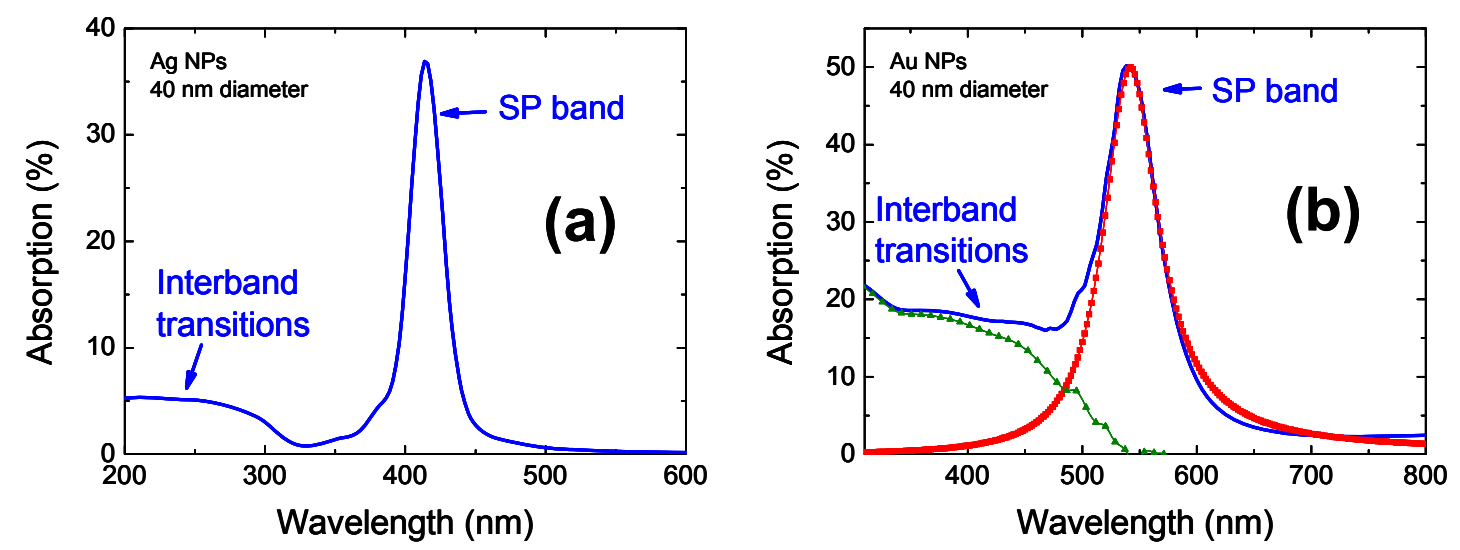

Figure 5. Optical absorption spectra for (a) Ag and (b) Au NPs with $40 \mathrm{~nm}$ size (embedded in a silica matrix with $\varepsilon=2.25)$. For Au NPs, the contributions to the optical absorption of interband transitions and SP are resolved.

The reason for this surprising behaviour can be qualitatively explained with the same classical picture of SP. When the incident light reaches the NP, the conduction electrons move resulting in a charge accumulation at the NP surface. This charge creates a field inside the NPs (the restoring field) but also out of the NP. The large electron density and mobility in the metallic NP produces a large charge accumulation at the NP surface and consequently intense fields in a region fairly larger than NP size. As figure 6 illustrates, there are wide regions were the electric field created by the particle is opposite to that of the light, so the interference results destructive, leading to light extinction beyond the NP volume [46]. For other regions, the result of the interference between both electric fields is a net field with other propagating direction, hence inducing light scattering. This mechanism explains qualitatively the huge extinction cross section of metallic NPs when SP are excited. Actually, the excitation of SP with laser sources can even yield a modification of the NP shape by local increase of the temperature associated to the huge absorption $[47,48,49,50]$.

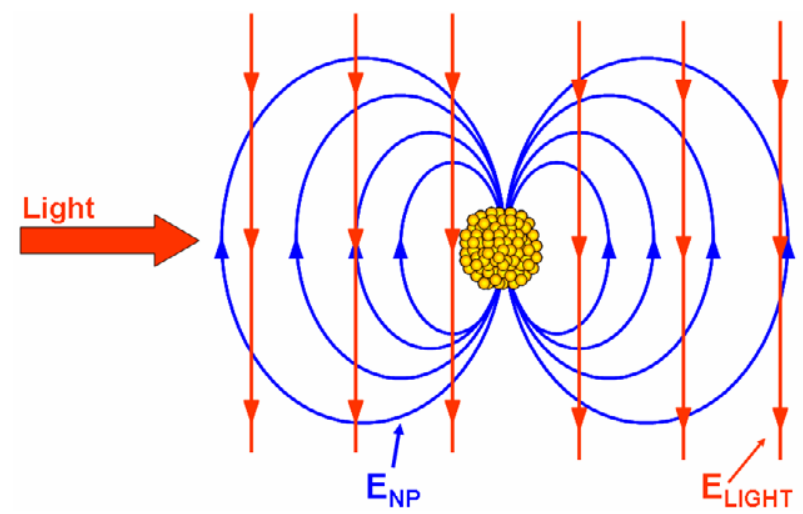

Figure 6. Illustration of electric fields of incident light and that created by the electron oscillations near the NP. 


\subsection{Models and calculations of SP}

While the above classical description can be useful to illustrate and understand what are SP, it is too simple to carry on numerical analyses. An accurate calculation of the SP and the associated light absorption requires solving the Maxwell equations at the NP region using the proper boundary conditions. The analytic solution can be obtained only for certain geometries and it was developed by Gustav Mie in the early $1900[1,26]$. The Mie theory provides an exact solution for spherical NPs assuming that they are non-interacting (i.e., the distance between the NPs is large enough so we may assume that the electric field created by a NP does not affects to the rest of them). In this case, Maxwell equations can be solved analytically and the extinction cross section is given by:

$$
\sigma_{e x}=\frac{2 \pi}{\left|k^{2}\right|} \sum_{L=1}^{\infty}(\mathbf{2} L+\mathbf{1}) \cdot \operatorname{Re}\left[a_{L}+b_{L}\right]
$$

being

$$
a_{L}=\frac{m \Psi_{L}(m x) \cdot \Psi_{L}^{\prime}(x)-\Psi_{L}^{\prime}(m x) \cdot \Psi_{L}(x)}{m \Psi_{L}(m x) \cdot \eta_{L}^{\prime}(x)-\Psi_{L}^{\prime}(m x) \cdot \eta_{L}(x)} \quad b_{L}=\frac{\Psi_{L}(m x) \cdot \Psi_{L}^{\prime}(x)-m \Psi_{L}^{\prime}(m x) \cdot \Psi_{L}(x)}{\Psi_{L}(m x) \cdot \eta_{L}^{\prime}(x)-m \Psi_{L}^{\prime}(m x) \cdot \eta_{L}(x)}
$$

Where $k$ is the light wavevector in the dielectric medium, $x=|k| \cdot R, R$ is the NP radius, , $m=n / n_{m}$ being $n$ and $n_{m}$ the refraction index of the metal (complex) and that of the surrounding dielectric medium respectively, and $\Psi_{\mathrm{L}}$ and $\eta_{\mathrm{L}}$ are the cylindrical Bessel-Ricatti functions. These equations are not easy to handle, although there are codes available to carry on calculations.

There is a large list of theories that allow calculating the light absorption associated with SP for specific size ranges or when NPs are not spherical or they are interacting. An excellent review of these theories is given in [1]. We survey here just the most common ones.

\section{The dipolar approximation}

The dipolar approximation offers simple equations to calculate light absorption of metallic NPs provided that the NP size is fairly smaller than the light wavelength (besides the hypothesis of Mie theory). As the SP resonance band falls at the visible part of the spectrum ( 360-720 nm), the condition of small NPs is usually achieved for NPs diameter below $\sim 50 \mathrm{~nm}$. In this case, the electric field inside the NP can be considered as uniform (see figure 7) and the particle can be described by an electric dipole. Mathematically, this approximation is achieved assuming that $\mathrm{x}$ is 
small enough, so, in equation (2) we can just consider the first term $L=1$. (Note that the term $L=0$ corresponding to the total electric charge of the NPs is zero).

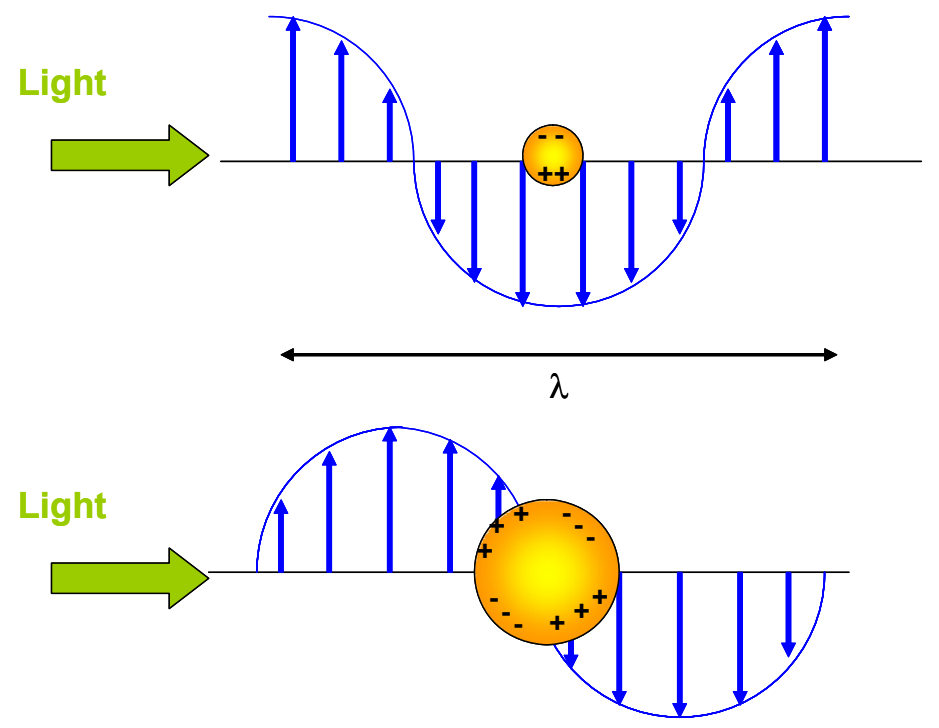

Figure 7. Electric field and charge distribution at the NPs surface for size (top) much smaller and (down) comparable to the light wavelength.

Within this approximation, the absorption cross section is given by:

$\sigma_{e x t}=\frac{24 \pi^{2} R^{3} \varepsilon_{m}^{3 / 2}}{\lambda} \frac{\varepsilon_{2}}{\left(\varepsilon_{1}+2 \varepsilon_{m}\right)^{2}+\varepsilon_{2}^{2}}$

As the NPs size increases, the approximation is not valid anymore, since the electric field inside the NP can not be considered uniform. In that case, more multipolar terms (i.e. more terms in equation 2) must be considered as discussed below.

\section{The Effective Dielectric Function Theories}

Another approach to calculate the optical properties of metallic NPs consist on replacing the heterogeneous system formed by the NPs (described by a complex dielectric function $\varepsilon_{1}+i \varepsilon_{2}$ ) and the surrounding media $\left(\varepsilon_{\mathrm{m}}\right)$ by a homogeneous medium with an effective dielectric function $\left.\varepsilon_{\text {eff } 1}+i \varepsilon_{\text {eff } 2}\right)$. These theories are known as effective medium theories. There are a large number of these theories depending on the exact conditions that are imposed to the homogeneous medium [1]. The first approach was proposed by Newton [1,51] defining an effective dielectric function as an average of the NPs and medium dielectric function weighted by their volume. 
However, the best approach within the framework of effective medium theories was developed by Maxwell-Garnett $[52,53,54,55]$ proposing to replace the NPs-dielectric medium system by an homogenous material exhibiting the same dielectric polarization upon light illumination. With this condition, the complex effective dielectric function is given by:

$$
\frac{\varepsilon_{\text {eff }}-\varepsilon_{m}}{\varepsilon_{\text {eff }}+2 \varepsilon_{m}}=f \frac{\varepsilon-\varepsilon_{m}}{\varepsilon+2 \varepsilon_{m}}
$$

and the extinction cross section of the material is then given by:

$$
\alpha\left(\mathrm{cm}^{-1}\right)=\frac{8.88 \cdot 10^{7}}{\lambda(\mathrm{nm})} \sqrt{-\varepsilon_{1 e f}+\sqrt{\varepsilon_{1 e f f}^{2}+\varepsilon_{2 e f f}^{2}}}
$$

being $\lambda$ the light wavelength. For the case of spherical, small and isolated NPs, this model leads to the same result that the dipolar approximation. An interesting advantage of the MaxwellGarnett model is that it can be modified to take into account interparticle interactions $[56,57]$ or the presence of non-spherical NPs $[1,58,59]$.

\subsection{Size effects}

NPs size has a dramatic effect on the SP resonance processes and consequently on the optical properties of the NPs. Actually, there are different mechanisms inducing size dependence of the SP properties. The predominant one will depend on the NP size range we consider. As a general approach, we may distinguish two regimes corresponding to small NPs (fairly smaller than the light wavelength) and large ones with size comparable to the wavelength.

Small NPs (up to $\sim 50 \mathrm{~nm}$ )

For this size range, we may assume that the NP is properly described by a dielectric dipole. The size dependence of the SP affects mainly the width and the intensity of the resonance band while the effect on the resonance wavelength is quite reduced [60]. Dealing with size effects in this range, we can distinguish between intrinsic and extrinsic size effects.

The intrinsic effects are related to the damping of the electron oscillations. When SP are excited, the electrons are damped in their movement by the scattering with the ionic cores and with the surface. The damping constant for the electron oscillations is given by:

$\gamma=\gamma_{0}+A \frac{V_{F}}{R}$ 
The first term $\left(\gamma_{0}\right)$ describes the damping due the scattering of the oscillating electrons with the ionic cores. It is size independent and its value is given by $\gamma_{0}=\mathrm{v}_{F} / l_{\infty}$, being $\mathrm{v}_{\mathrm{F}}$ the velocity of the conduction electrons (Fermi velocity) and $I_{\infty}$ the electron mean free path in the metal. This term depends just on the nature of the metal and the crystal structure. For Au at $300 \mathrm{~K}$ we have $V_{F}=1.4 \cdot 10^{6} \mathrm{~m}^{-1}$ and $\mathrm{I}_{\infty}=30 \mathrm{~nm}$ leading to $\gamma_{0}=4.6 \cdot 10^{13} \mathrm{~s}^{-1}$ and for Ag we have $V_{F}=1.39 \cdot 10^{6} \mathrm{~m} \cdot \mathrm{s}^{-1}$ and $\mathrm{I}_{\infty}=52 \mathrm{~nm}$ leading to $\gamma_{0}=2.6 \cdot 10^{13} \mathrm{~s}^{-1}$.

The second term corresponds to the scattering of oscillating electrons with the particle surface. A is a material dependent constant that takes into account the features of the surface scattering. It is commonly considered as a phenomenological parameter although it is well established that it depends on the surface features as crystal quality, facets, capping species, strain, etc $[1,60]$. The electrons in a certain shell close to the NP surface will scatter this surface when they oscillate. The larger the velocity of the oscillating electrons (which is about the Fermi velocity), the larger the number of electrons scattering the surface during their movement (i.e., the thickness of the shell of electrons scattering the surface during oscillations). As the particle size increases, the fraction of electrons in the shell close to the surface decreases and therefore the total damping is reduced. Therefore, the surface damping results proportional to the Fermi velocity in the metal and inversely proportional to the particle radius.

It is well known that the resonance of a linear oscillator reduces its intensity and increases the width with the damping constant, while the effect on the resonance frequency is very limited (a slight shift toward smaller frequencies, i.e. larger wavelengths). Experimentally, it is observed that the full-width-at half maximum (FWHM) of the SP resonance band is given by:

$\Gamma=\mathrm{a}+\frac{\mathrm{b}}{\mathrm{R}}(7)$

Figure 8 presents the optical absorption spectra of silver NPs embedded in silica $(n=1.5)$ with different size. The resonance absorption intensity increases with particle size while the resonance peak slightly moves. The dependence of the FWHM of the resonance peak with the particle size, shown in figure 8 follows the inversely proportional law of eq. 7 for radius below $25 \mathrm{~nm}$ (inset in figure 8). 

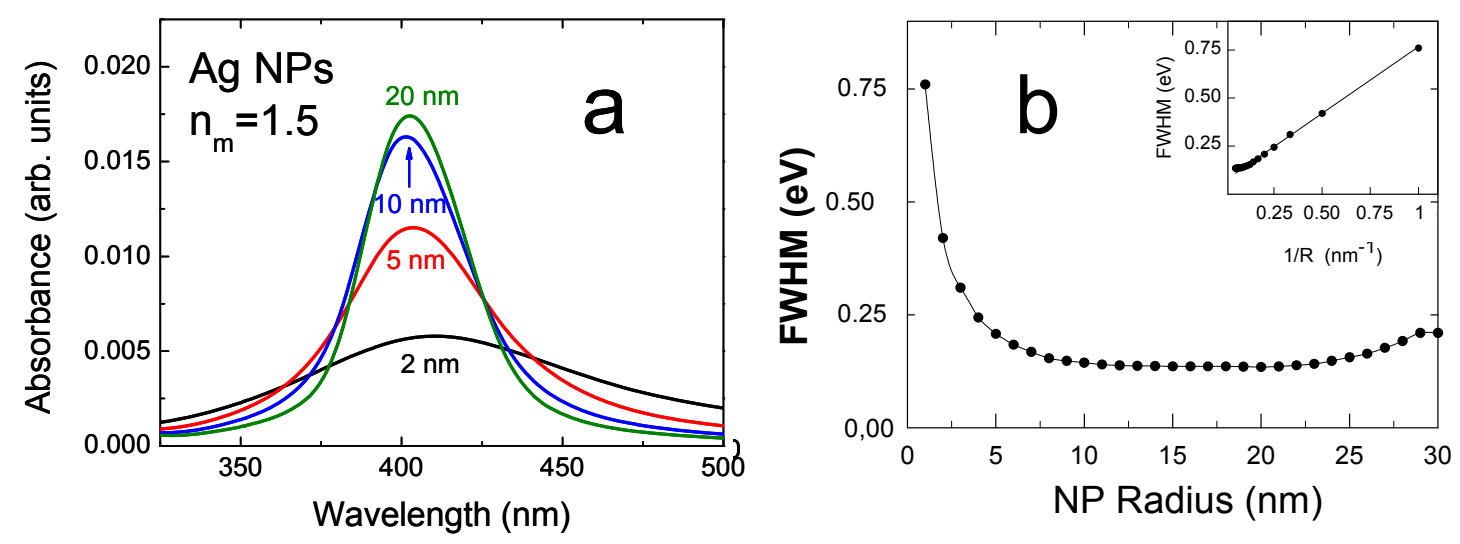

Figure 8 (a) Optical absorption spectrum for Ag NPs with different size calculated according to the Mie theory. (b) FWHM as a function of the particle radius; inset shows the linear relationship between FWHM and the inverse of the radius.

Besides this intrinsic effect of the NPs size on the SP, it has been reported that the dielectric function of small NPs is also size dependent $[1,61]$. At this scale, the energy bands are not so well defined as in a bulk solid, and consequently contribution of the interband transitions to the dielectric function results size dependent. Consequently there is an additional dependence of the SP on the NPs size through the dependence of its dielectric function. This effect is also appreciable for NPs below $5 \mathrm{~nm}$ size and the value of the dielectric function can be calculated from the bulk one [61].

Large NPs (above $\sim 50 \mathrm{~nm}$ )

NPs with radius larger than $50 \mathrm{~nm}$ can not be considered anymore much smaller than the light wavelength inside the metal. Thus, the particle is no more described by a dipole (figure 7) and further multipolar terms are required. As a consequence, the resonance band splits into several peaks: two peaks for quadrupole, three peaks for a octopole, etc [60]. Figure 9 shows the contribution of the different terms in equation 2 to the extinction cross section of Au NPs.

For Au NP with radius below $60 \mathrm{~nm}$, the quadrupolar term $(L=2)$ is negligible and the multipolar effects must be taken into account. 


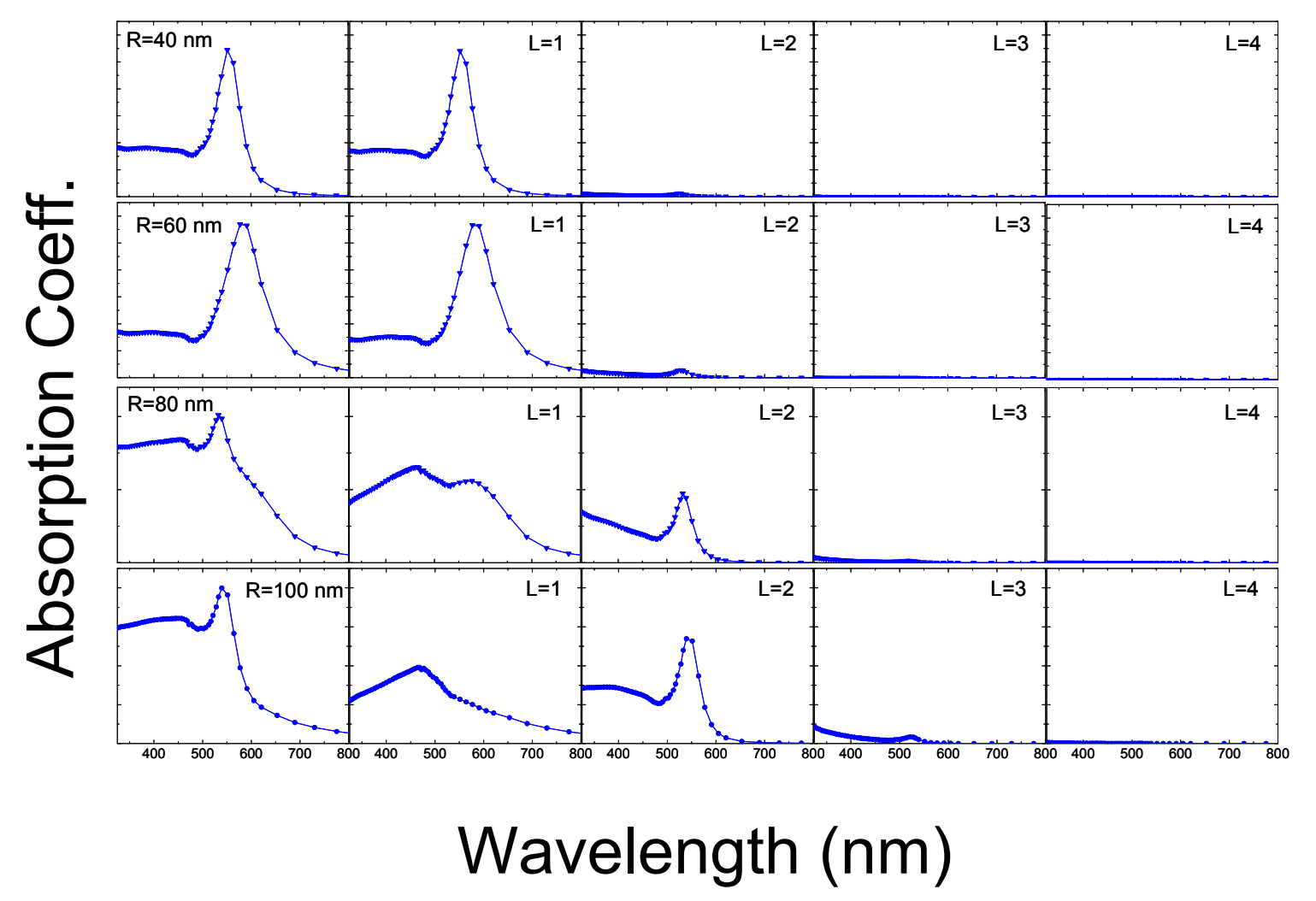

Figure 9. Optical absorption spectrum for Au NP with different size calculated according to the Mie theory for $n_{m}=1.5$ and the contribution of the different multipolar terms

If the NP size is further increased, NPs can not be considered anymore as multipoles and the SP become propagating waves with well defined modes or dispersion relations [2]. These are named itinerant SP and represent a quite different kind of phenomenology [3].

\section{Size dispersion}

Although synthesis methods for metallic NPs improved substantially in the last years, the result is always a set of NPs with certain size dispersion. Consequently, the experimentally measured optical absorption spectrum of a material containing metallic NPs, will be a weighted average of the absorption spectra corresponding to the NPs present in the sample.

The size dispersion induces a broadening of the absorption band. As figure 10 illustrates, the larger the size dispersion, the wider the absortion band. In particular, we found that for a set of $\mathrm{Ag}$ NPs with average size of $10 \mathrm{~nm}$ and a dispersion $\pm 4.2 \mathrm{~nm}$, the FWHM of the SP absorption band is three times smaller than that for $10 \mathrm{~nm}$ Ag NPs with a size dispersion $\pm 8.5 \mathrm{~nm}$. Thus, it is not possible to determine the size of the NPs just from the FWHM of the absorption band, as it depends on two parameters: average size and size distribution width. 


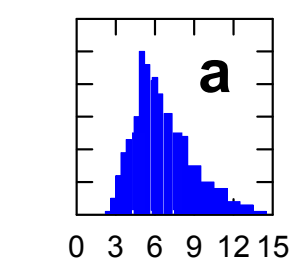

\section{Radius (nm)}

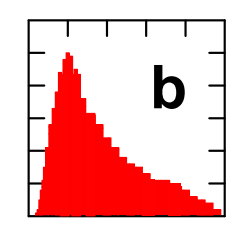

$0 \quad 510152025$

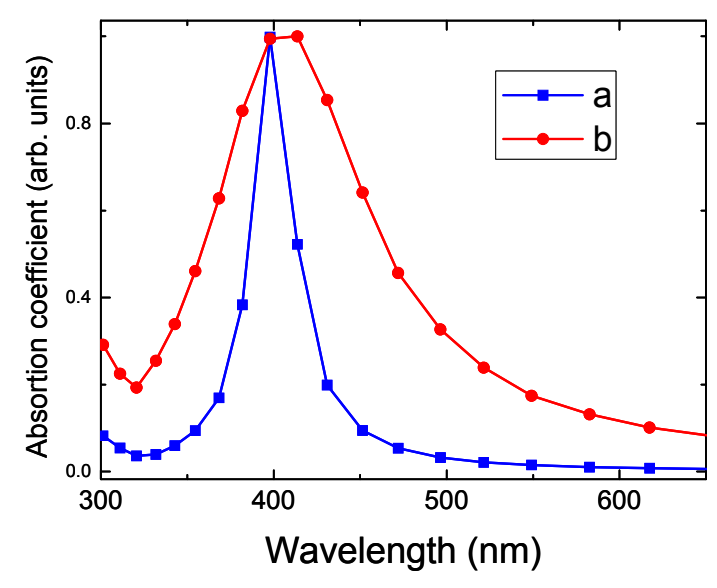

\section{Radius (nm)}

Figure 10 (left ) size distributions and (right) optical absorption spectra for Ag NPs both with $10 \mathrm{~nm}$ average size but different size dispersion calculated according Mie theory and using $n_{m}=1.5$. For size distribution (a)

\subsection{Shape effects}

The resonance of SP is strongly affected by the particle shape. Since the restoring force for SP is related to the charge accumulated at the particle surface, it will be influenced by the particle geometry. The most clear example of these shape effect are the nanorods [59,62]. As figure 11 illustrates, for a nanorod, the charge accumulation at the NP surface will be different for electron oscillations along the rod axis (longitudinal plasmons) and or along perpendicular direction (transversal plasmons). In particular, the charge accumulation will be maximum for this later and minimum for electron displacement along the rod axis. The restoring force is proportional to this charge accumulation, and therefore, for electrons oscillating along the rod axis we should expect smaller forces and consequently smaller resonance frequencies (i.e., larger resonant wavelengths). For nanorods, the resonant frequency of transversals plasmons falls at about the same position that for spherical NPs (actually, at wavelengths slightly smaller) while the resonance of longitudinal plasmons shifts toward larger wavelengths when the nanorod aspect ratio increases, as illustrated in figure $11 \mathrm{~b}$. This behaviour provides a method to tune the resonance of the SP at the desired wavelength by controlling the aspect ratio, which is especially useful for biomedical purposes as explained in section 3.1.

Using polarised light it is possible to excite separately the longitudinal or transversal SP of a nanorod. However, if the rods are not macroscopically oriented (as it is the case for colloidal 
suspensions or solutions) we will always observe an average over the possible orientation with both, longitudinal and transversal resonance bands in the spectrum as in figure $11 \mathrm{~b}$.

Other geometries as triangular prisms, nanocubes or nanocages give rise to more complicated effects, but in general [59], deviation from sphericity shifts the resonance toward larger wavelengths.

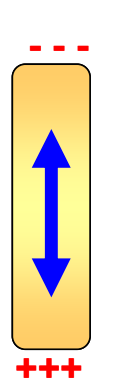

\section{(a)}

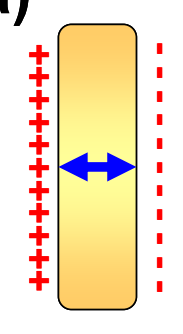

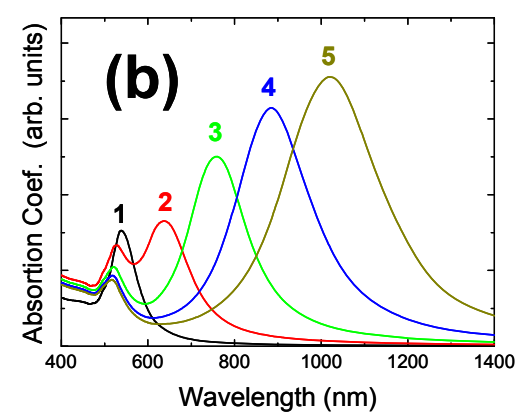

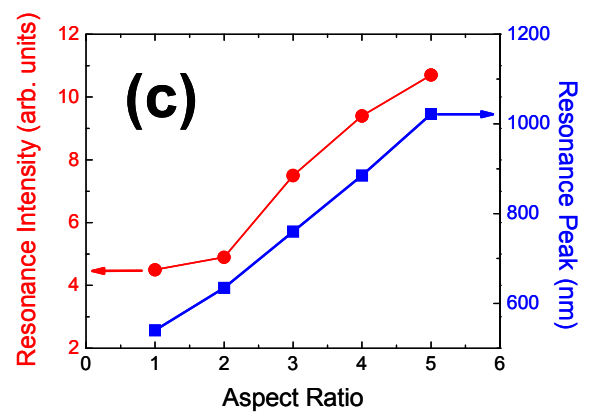

Figure 11. (a) Illustration of charge accumulation for longitudinal and transversal SP (b) Calculated absorption spectra for Au nanorods with different aspect ratio indicated in the figure. (c) Resonant position and intensity of the longitudinal SP as a function of the nanorod aspect ratio.

Calculations for non spherical NPs are rather complicated. Actually the Maxwell equations can be solved analytically only for spherical nanoshells or ellipsoids. The Gans theory $[1,58,59]$ provides a method to calculate the SP spectra of non spherical NPs within the dipolar approximation framework by approximating the nanostructure to an ellipsoid.

For other geometries, calculation of the SP spectra can be addressed by the so-called discrete dipole approximation $[59,63]$. Within this method, the nanostructure is replaced by an ensemble of nanospheres fairly smaller than the nanostructure. Each nanosphere is described by a polarization factor (than can be derived from Maxwell-Equations since they are assumed to be spherical) and the field at the nanosphere position is that of the incident light plus the field created by the rest of NPs. With this method, any geometry can be in principle addressed.

\subsection{Surrounding media}

The excitation of SP does not depend only on the NPs features but it is also significantly modified by the surrounding medium. It has two clear effects on the SP excitation process. On the one hand, the dielectric function of the surrounding medium determines the light wavelength at the vicinity of the NP hence altering the geometry of the electric field at the NPs surface. 
But the most important effect is related to the polarization of the medium. During SP excitation, the charge accumulation creates an electric field in the vicinity of the NPs (beside that of the incident light). This field induces the polarization of the dielectric medium, resulting in a charge accumulation at the edges of the medium (i.e., at the interface between the dielectric and the metallic NPs) that will partially compensate the charge accumulation due to the conduction electrons movement. This reduction of charge will depend on the dielectric function of the media; the larger $\varepsilon_{\mathrm{m}}$, the larger the polarization charge, and hence, the larger the effect on the SP. Reducing the net charge at the NP surface implies a reduction of the restoring force. For an oscillator, it is well known that reducing the restoring force leads to smaller resonant frequency. Thus, increasing the dielectric constant of the surrounding media will shift the SP resonant band toward larger wavelengths (i.e., smaller frequencies).

Figure 12 shows the absorption spectra for 10nm size Au NPs embedded in media with different dielectric functions. The surrounding medium has a limited effect on the bandwidth (measured in $\mathrm{eV}$ ), that is mainly related to the particle size. The resonance intensity also increases with the dielectric function of the medium. This is the expected behaviour for a forced oscillator: when the resonance frequency decreases, the amplitude at resonance increases. This is mathematically reflected in eq. 3 , where the absorption cross section is proportional to $\varepsilon_{m}^{3 / 2}$.

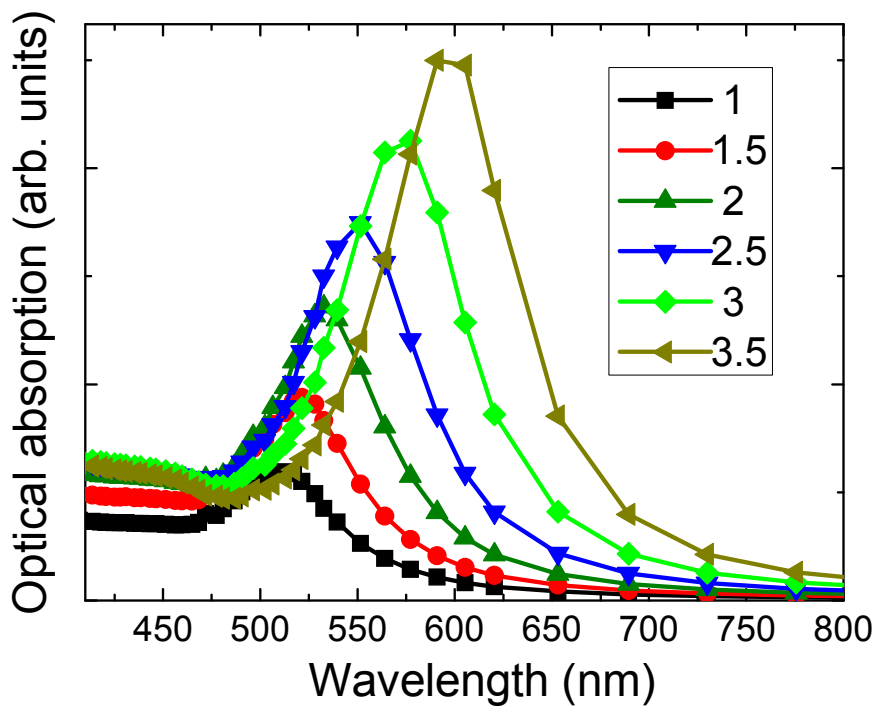

Figure 12 Optical absorption spectra for Au NPs with $10 \mathrm{~nm}$ size in a dielectric medium with different dielectric function calculated according to Mie theory. 
NPs obtained by chemical routes are commonly capped with organic molecules to prevent agglomeration. These capping agents have a significant effect on the SP features since they represent the very first environment of the NP. Moreover, the strength of the bonds between the NP and the molecules induces an extra effect $[1,64,65]$. The modification of the NP electronic configuration due to surface bonds modifies the conditions for electron oscillation, yielding to variations in the SP spectrum that can be used to investigate the characteristics of these bonds [66].

\subsection{NPs interactions effects.}

As detailed above (see figure 6), the excitation of SP induces an electric field in the vicinity of the NPs. If the NPs are close enough, the net applied field at the NPs positions will be that of the incident light plus the field created by the rest of NPs. Therefore, the resonant conditions will be modified. In general, interparticles interaction red-shift the resonance band increasing the FWHM as illustrated in figure $13 a[56,57,67,68]$. When NPs are arranged in particular geometries it is possible to create controlled interferences that allow additional tuning of the SP excitation band (figure 13b). These effects can be achieved when the NPs are created by film deposition and patterning or by self-assembly processes.
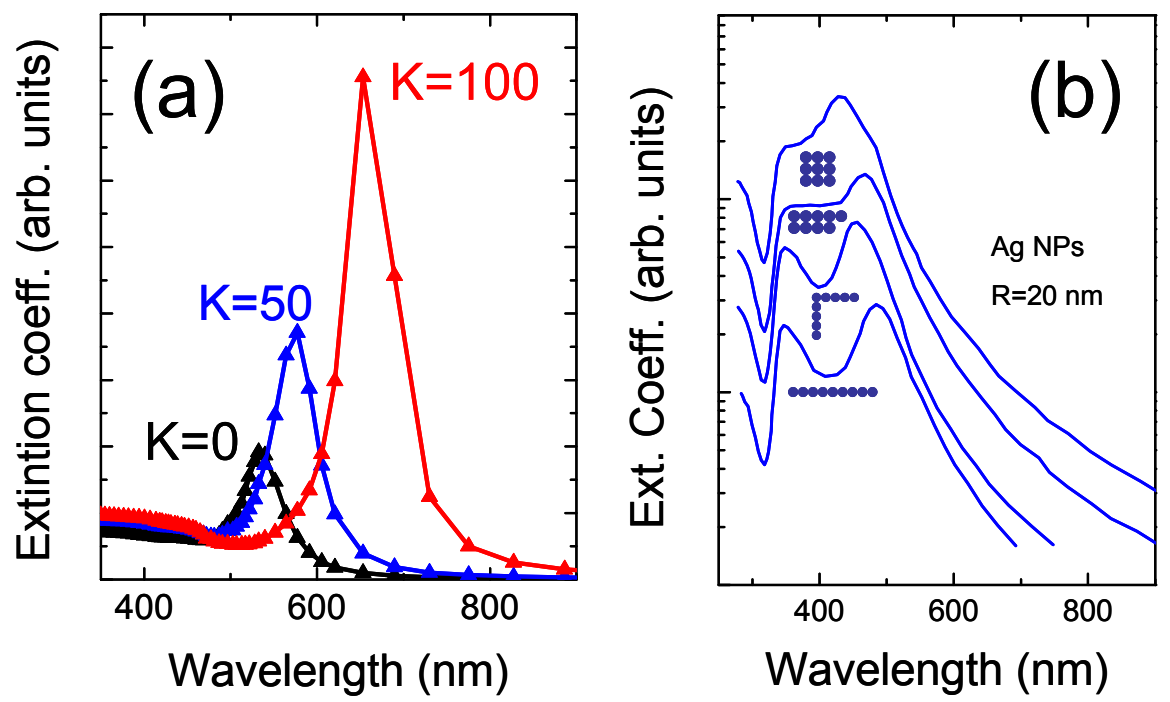

Figure 13. (a) Optical absorption spectra for Au NPs with dipolar interactions. Parameter K represents the intensity of the electric field created at a NP position by the rest of the NP as described in [57] (b) Optical absorption spectrum of arrays of $20 \mathrm{~nm}$ Ag NPs with different geometries. Adapted from [1].

The field created by a NP quickly decays with distance and therefore, the main effect is due to adjacent NPs while those far away have a very weak effect (note that for a dipole, the electric field decreases as $\left.1 / \mathrm{r}^{2}\right)$. Due to this fast decay, the electric field created at a NP position by other 
NPs close enough, will not be uniform but it will exhibit significant amplitude variations in the NP volume. As SP resonance is a resonant process, it is required that all the electrons receive the same electric field; otherwise the electrons movement will be heterogeneous leading to charge accumulation inside the NP volume that rise electric forces, resulting on a extra damping of the oscillations.

\section{Applications}

The high absorption coefficient associated with the excitation of SP opens a wide range of potential applications of metallic NPs in many fields. The possibility to concentrate, amplify and manipulate light at the nanoscale through SP, provides a method to improve optical effects or activate processes in a controlled way. There are hundreds of potential applications of SP in different fields that have been thoroughly reported in literature impossible to review here. We will just highlight some of them in order to illustrate capabilities of SP in four selected fields: biomedicine, energy, environment protection and information technologies.

\subsection{Biomedicine}

Biomedical applications are certainly the most developed SP potential uses. Besides the possibility to explode SP, noble metal NPs exhibit additional features that make them specially useful for this field $[59,69,70]$. NPs have the biological size, comparable to entities as virus, DNA chains, cells or bacteria. Hence, it is possible the NPs to interact individually with these organisms increasing efficiency and specificity of medical treatments. Moreover, noble metal NPs are highly biocompatibility and easy to functionalize (mainly through thiol chains that create strong bonds with NPs surface and may act as linkers with larger organic molecules). Thus, in vitro applications of metallic NP exploding SP are well established while in vivo ones (always slower to develop due to the requirements of long term effect essays) show certainly promising results.

\section{DNA sequencing}

Noble metal NPs are nowadays used for DNA sequencing, replacing the dyes based technology $[71,72]$. The use of dyes causes some problems as photobleaching and instability in certain environments that limit their applications [72,73]. Moreover, the spatial resolution and sensitivity required to carry out thousands of test compel to use confocal microscopy leading to complex and expensive devices [74]. The use of metallic NPs may overcome these limitations as noble 
metals are highly stable and the huge absorption cross section associated with SP resonance allows the easy detection of small quantities of NPs.

Figure 14 illustrates the strategy for the development of DNA sequencers. The target DNA chain to be analyzed is attached to a metallic NP (gold NPs are the most commonly used ones) creating a NP/DNA complex (figure 14a) by polymerase chain reaction or through thiol chains $[72,75]$. A sensing surface is functionalized with patterns of oligonucleotides (short nucleic acid chain, typically with fifty or fewer bases) to be used as probes (figure 14b). These oligonucleotides are able to form self-assembled monolayers prompting the formation of geometrical dense patterns. Finally, the solution containing the target NP/DNA complex is placed over the sensing surface. DNA strands spontaneously bind to oligonucleotides containing the complementary chain [76]. Therefore, the target DNA/NP complexes will accumulate over the surface functionalised with oligonucleotides with chains complementary to the target DNA one (figure 14c). Illuminating the sensing surface with light at the plasmonic frequency of the gold NPs, it is possible to detect the regions where the DNA/NPs complexes are accumulated. The large scattering due to SP excitation allows detecting small amounts of NPs in small regions using an optical microscope (figure 15). Sensing surfaces with a large number of oligonucleotides can be automatically analysed with a digital camera, allowing a quick identification of the sequences present in the target DNA [72] as illustrated in figure 15.

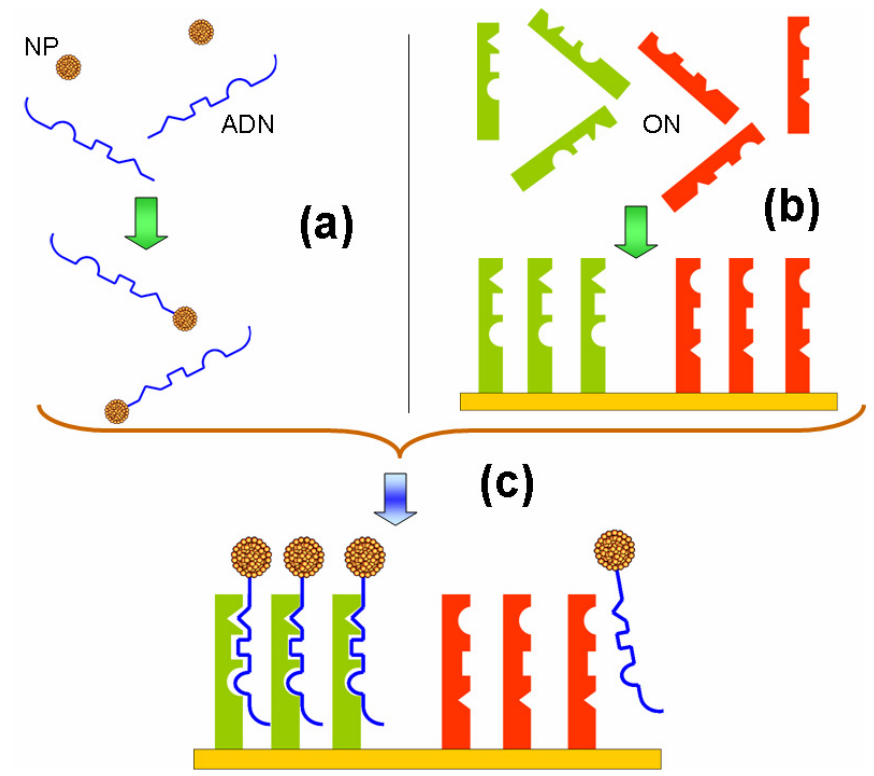

Figure 14. Scheme of the DNA labelling process with NPs.(a) Target DNA chains are attached to metallic NPs. (b) A sensing surface is prepared with regions of oligonucleotides (ON) with specific sequences to be detected. (c) When the solution containing DNA/NPs complexes is placed on the sensing surface, these complexes will bind to the oligonucleotides with the sequences complementary to the target DNA; the SP allows the spatial detection of the NPs identifying the sequences present in the target DNA. 


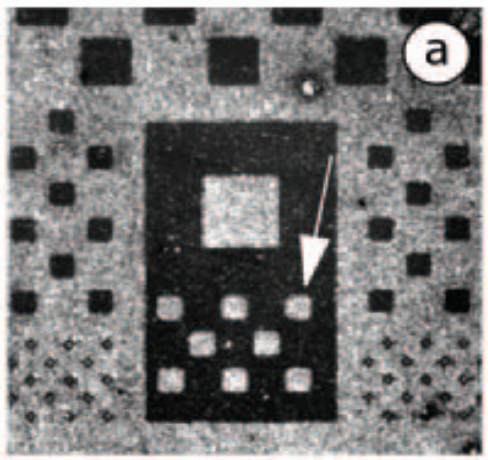

$10 \mu \mathrm{m}$

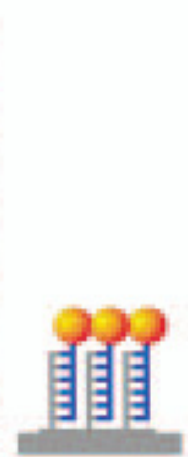

0

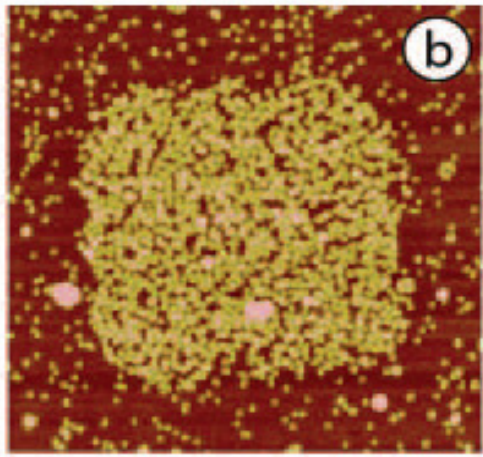

50

$1 \mu \mathrm{m}$

Figure 15. (left) Optical reflection image of NP-labelled DNA chip; the regions with NPs appear brighter due to the reflection of incident light. (right) Zoom of one square by scanning force microscopy reveals individual NPs. Reproduced with permission from [72].

\section{Cell labelling}

Following a very similar strategy, it is possible to use SP for cell labelling. To this purpose, NPs are functionalized with biomolecules exhibiting affinity for certain species and/or particular cell features and introduced into cell cultures. This technology is particularly useful to identify cancer cells $[77,78,79,80,81]$. NPs functionalized with molecules having higher affinity for these cancer cells than for normal ones, will concentrate around these cancer cells that can be then detected by optical microscopy.

Au NPs can be used to label malignant epithelial cells as demonstrated by El-Sayed et al [80]. Non-functionalised NPs tend to accumulate within the cell cytoplasm providing anatomic labelling information of the cell. The NPs exhibit the same affinity for malignant and non malignant cells. However, if the NPs are functionalized with monoclonal anti-epidermal growth factor receptor antibodies, the affinity for malignant cells is 6 times larger than for non-malignant ones. The NPs accumulation is detectable with optical microscopy at SP wavelength due to the high scattering cross section, providing a method to identify malignant cells specifically (figure 16). In addition, a spectroscopic study have shown that the SP absorption band for the NPs accumulated at the surface of malignant cells results sharper and red shifted [80], which may be an additional method for cell labelling using smaller quantities of NPs and more reliable than intensity contrast. 


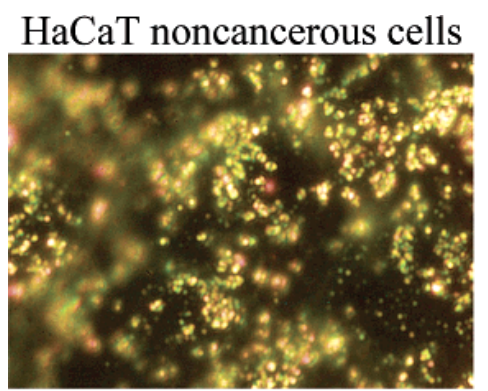

HOC cancerous cells

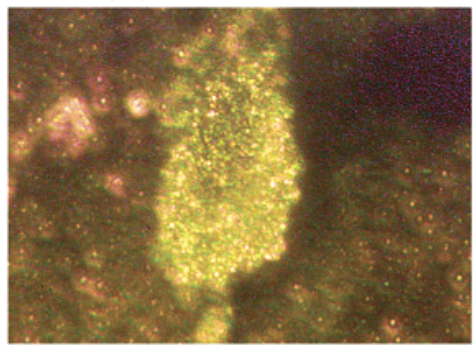

HSC cancerous cells

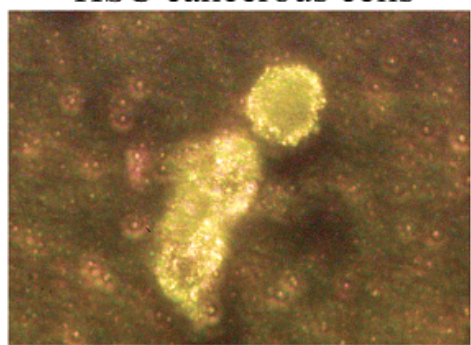

Figure 16. Light scattering images of HaCaT non-cancerous cells (left), HOC cancerous cells (middle), and HSC cancerous cells (column) after incubation with anti-EGFR antibody conjugated gold NPs. The conjugated NPs bind specifically to the surface of the cancer cells (right). Reproduced with permission from [80].

Similar works have demonstrated selective labelling using Au NPs functionalized for ductar carcinoma cells (ATCC: Hs 578T) [79], human epidermal growth factor receptor 2 (HER2) [82], cervical epithelial cancer cells ( $\mathrm{SiHa}$ ) [83] human oral squamous carcinoma cells ( $\mathrm{HOC}$ ) [84] and others $[85,86]$. Outstanding results have been also achieved by using combined techniques in which the excitation of SP is used to amplify or trigger other optical processes as photoacustic imaging [87] SERS [88,89] or dye fluorescence [90].

\section{In vivo labelling and imaging}

Development of in vivo imaging and labelling techniques are quite limited because the absorption band of SPR is almost identical to that of haemoglobin, the main protein of human blood as shown in figure 17. Therefore, it is very difficult to detect or energize gold NPs in the bloodstream.

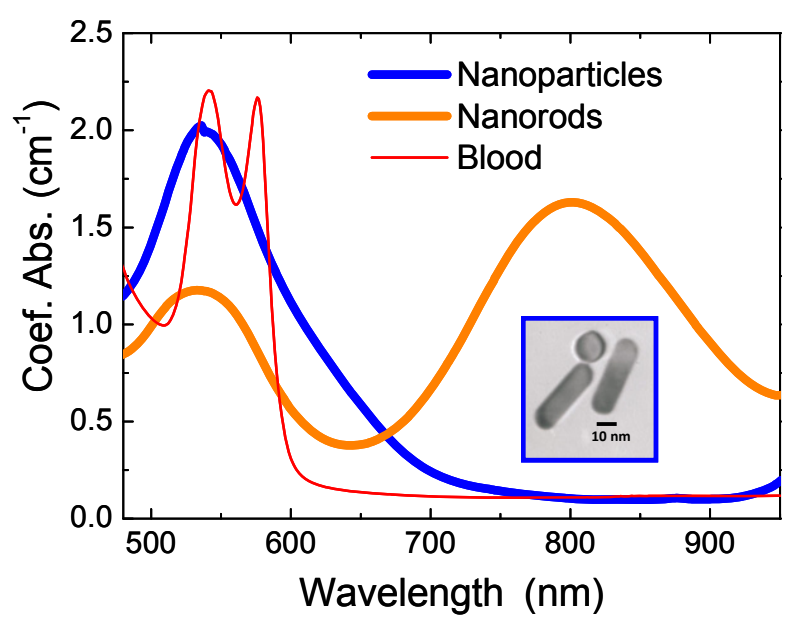

Figure 17. Optical absorption spectrum of Au NPs and nanorods. Inset shows a TEM image of the nanorods. Absorption spectrum of human blood is also shown for comparison. While the SP band of NPS and the transversal SP of nanorods falls in the same region of the spectrum that the blood, the longitudinal SP band is located at the Near-IR, where biological tissue scarcely absorbs. 
The strategy to overcome this limitation is the use of non-spherical NPs such as nanorods, nanocubes, nanocages $[59,74,81,91]$. Among these geometries nanorods are the most commonly used ones since controlling their aspect ratio it is possible to tune the SP resonance position to the "biological window" between 800 and $110 \mathrm{~nm}$ where the blood and tissues scarcely absorbs (see figure 17). Nevertheless, even with the use of nanorods, it is very complicated to observe directly scattered or transmitted light across biological tissue due to the large number of scattering bodies in the bloodstream (erythrocytes, leukocytes, thrombocytes, etc). Thus, the best results in this line up to now have been achieved by using nanorods combined with other techniques as SERS [92] or photoacoustic imaging.

For the combined use of SP resonance and photoacoustic technique, gold nanorods functionalised with the appropriated biomolecules are injected in the tissue. The tissue is later illuminated with pulses of light at the SP resonance wavelength in the NIR. The strong absorption cross section of the nanorods leads to a local increase of temperature during laser pulse. This heat is quickly dissipated to the adjacent tissue which undergoes a thermoplastic expansion creating an acoustic wave that propagates through the tissue and can be mechanically detected at the surface. Using this technique, Eghtedari et al recently detected Au nanorods in mice [93]. The experiment is illustrated in figure 18. Au nanorods where chemically synthesised controlling the aspect ratio to exhibit the maximum absorption of longitudinal SP at about $750 \mathrm{~nm}$. The nanorods were injected in the mouse that was placed onto an acoustic transducer to detect acoustic waves and convert them into an electrical signal. The mouse was illuminated with an alexandrite laser (757 nm wavelength) emitting $75 \mathrm{~ns}$ pulses with an average energy per pulse of $6 \mathrm{~mJ} / \mathrm{cm}$. The laser beam scanned the mouse recording the signal on the transducer after each pulse. Regions with high concentration of Au nanorods presented enhanced light absorption and consequently produced more intense acoustic waves after the laser pulse, that were detected with the acoustic transducer few microseconds after the laser pulse. The images of photoacoustic waves intensity obtained upon scanning the mouse with the laser beam before and after gold nanorods injection are shown in figure 18. The region where the nanorods were injected results brighter demonstrating the capability of the method to detect gold nanorods into a living organism. 

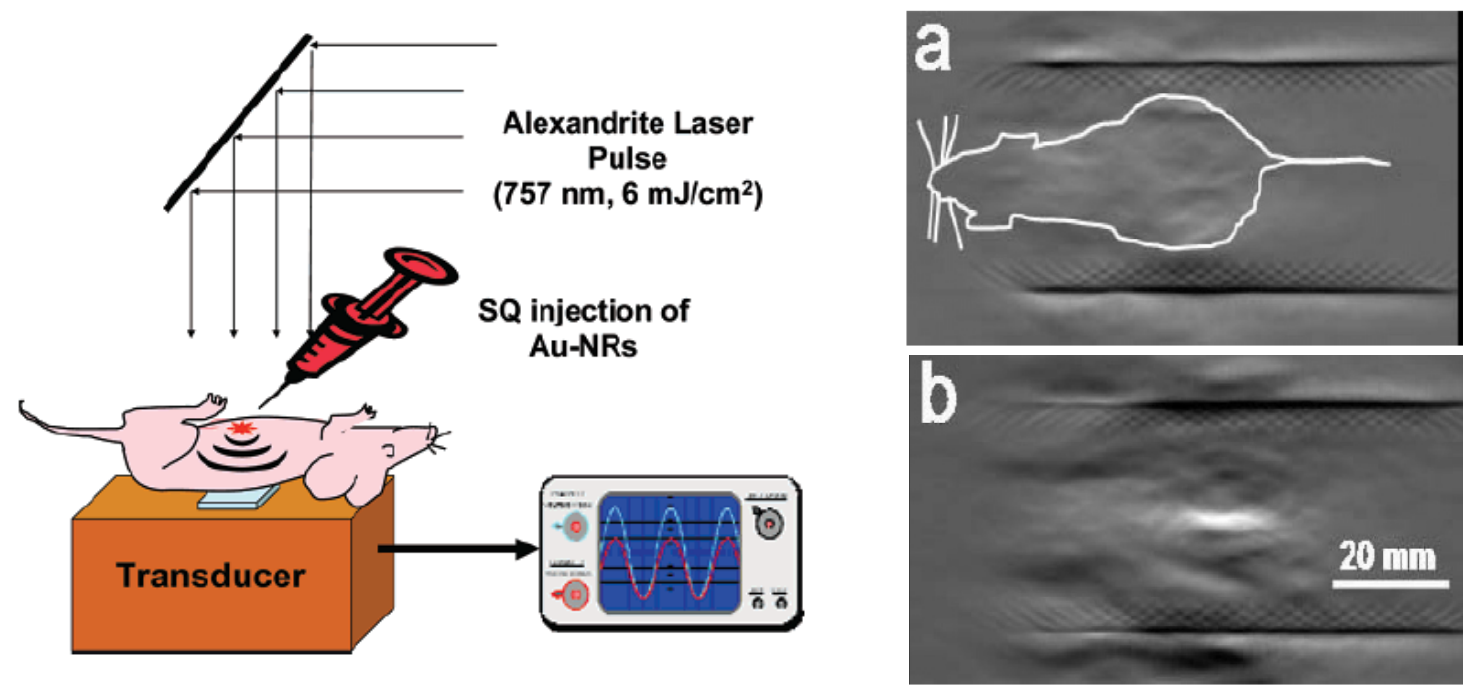

Figure 18. (left) Schematics of in vivo experiment to detect injected gold nanorods by optoacoustic imaging. (right) A typical optoacoustic image of a nude mouse before (a) and after (b) subcutaneous injection of Au nanorods in the abdominal area. Injected NPs were brightly visible in the optoacoustic image (b). Drawing in (a) depicts the approximate position of the nude mouse during experiment. Reproduced with permission from [93].

\section{Hyperthermia}

In addition to the possibility to detect, image and label biological entities, the SP offer the possibility to activate processes in a controlled way, so they can also be used for therapy. The intense light absorption and electromagnetic field enhancement allow energizing the NPs to trigger events locally as desired.

Hyperthermia is a non-invasive technique for cancer treatment consisting of heating the biological tissues to temperatures slightly $\left(\sim 5-10^{\circ} \mathrm{C}\right)$ over the normal one to promote the selective destruction of abnormal cells [94]. This increase of temperature may induce denaturalization of proteins, disruption of the organized biomolecular assemblies in the nucleus and cytoskeleton, and increase the membrane permeability [95]. A further step is the selective hyperthermia, consisting of a controlled heating of malignant cells. This strategy can provide advantages in cancer treatments as the normal tissue remains unaffected promoting the replacement of malignant cells with healthy ones, although the long term impact on patients is still undetermined. The light absorption associated to SP excitation produces a local heating that can be exploded for selective hyperthermia. To this purpose, nanorods are functionalized to bind specifically malignant cells (as in the imaging techniques described above). When the tissues are illuminated with NIR laser at the SP wavelength, the energy absorbed by the nanorods is partially converted 
into heat, increasing locally the temperature. Due to the high absorption efficiency of nanorods it is possible to increase their temperature several degrees. As the functionalization of the nanorods promote their accumulation around malignant cells, these later will be selectively heated, favouring their elimination with a reduced effect on healthy cells.

Recently, Wei et al $[, 95,96,97]$ shown in vitro selective elimination of adherent human KB cells (a tumour cell line derived from oral epithelium) using gold nanorods to perform localized hyperthermia. Adherent KB cells cultive were exposed to gold nanorods conjugated to promote the binding to the cell membrane (see figure 19).
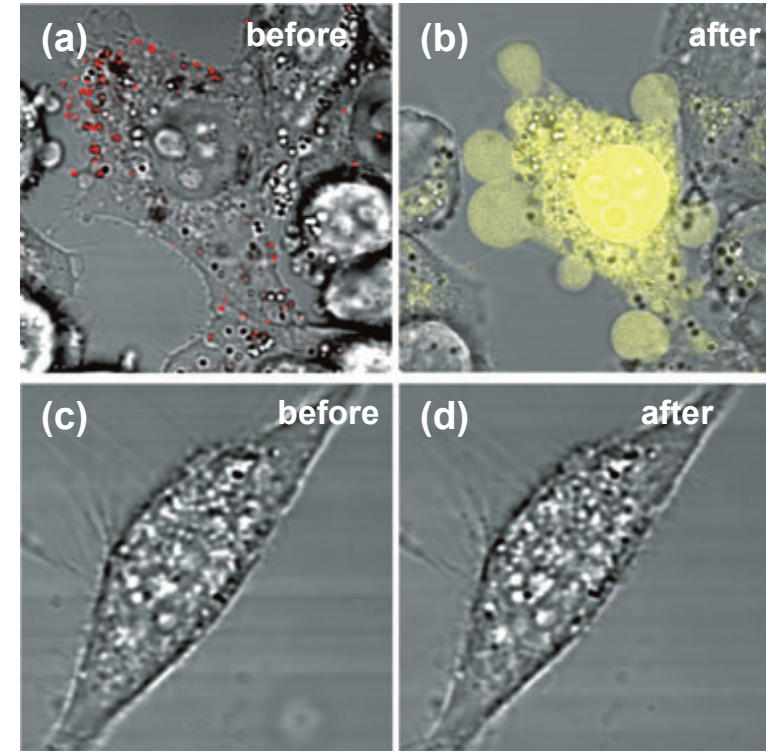

Figure 19. Photothermolysis mediated by folate-conjugated nanorods. (a, b) KB cells with membranebound nanorods (red) exposed to fs-pulsed near-infrared laser irradiation experienced membrane damage and blebbing. The loss of membrane integrity was indicated by ethidium bromide nuclear staining (yellow). (c, d) NIH-3T3 cells were unresponsive to nanorods and did not suffer photoinduced damage under the same irradiation conditions. Reproduced with permission from [96].

A laser working at $815 \mathrm{~nm}$ (where the NR exhibited a high absorption due to excitation of SP) with femtosecond pulses was used to irradiate the cultive. The irradiation induced a local heating of the nanorods (and consequently of the malignant cells) leading to a severe damage of the membrane. The membrane blebbing was optically confirmed by using a dye (ethidium bromide) as indicator of the membrane permeability. As figure 18 illustrates, in absence of nanorods, the cell did not suffer appreciable effects. Therefore, with the proper functionalization of the nanorods to bind specifically malignant cells, it is possible to perform selective hyperthermia on these cells, while normal ones do no suffer significant damage. Positive results have been found also 
functionalizing $\mathrm{Au}$ nanorods with CTAB and folate that promotes nanorods uptake and internalization by the KB cells instead of accumulating at the membrane [95].

\section{Drug delivery}

This heat dissipation and local increase of temperature associated with SP excitation can be also used for drug delivery. This is particularly useful for certain drugs that are highly aggressive and may induce undesired secondary effects on normal tissues. Controlled drug delivery leads to higher efficiency reducing these secondary effects. Commonly, the strategies of drug delivery are based on covering the drug with a coating that avoids interaction with non-targeted cells [98]. Once the drug reaches the target cell, the coating is removed releasing the drug to act at the desired region. Moreover, releasing rate can be also controlled to improve effectiveness. Metallic NPs holding SP can be used to this purpose $[99,100,101,102]$. A controlled release of the drug can be achieved by exciting SP: the light concentration around the NP can provide the energy required to activate the drug release process. Figure 20 presents an example of SP controlled drug delivery [98]. The drug is coated with a shell consisting on a polymer with embedded gold NPs and functionalized on the outer side with antibodies for specific cells. Once the ensemble reaches the target cells, the region is irradiated with light at the SP wavelength. The excitation of SP at the NPs induces a local heating and melting of the polymer shell, releasing the drug at the desired region and time. The heating efficiency of the metallic NPs can be optimized with different methods [103].

(a)

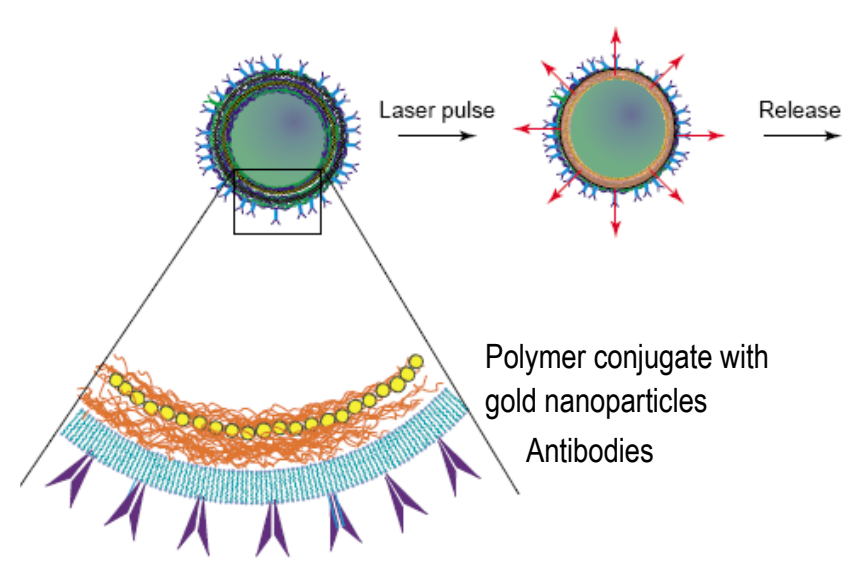

(c)

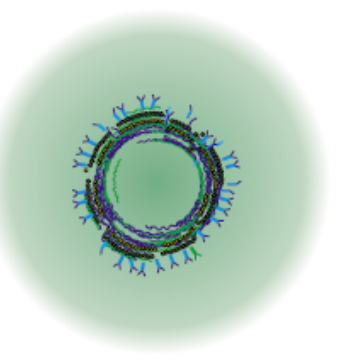

Figure 20. (a) Drug is coated with a polymer shell containing gold NPs and antibodies for specific uptake. (b). When the drug reachs the target, SP are excited with a laser beam, the dissipated heat melts the polymer and (c) release the drug at the desired region. Reproduced with permission from [98]. 


\subsection{Energy}

The nowadays energy problem impels to search for energy sources different to traditional ones based on fossil fuels. In this search, the sun appears as a clean source of energy with lifetime over 5000 million years releasing daily 4000 times the earth electric energy consumption. The use of solar energy can be addressed by different techniques including photovoltaic $[104,105,106,107]$, photochemical $[108,109,110]$ or photothermal $[111,112,113]$ ones. The main limitation of these technologies relays on the efficiency of the light absorption and conversion processes that make them hardly viable from an economical point of view. The capabilities to concentrate and locally amplify the light electric field by means of SP offer new methods to increase the efficiency of photoenergetic process, overcoming the efficiency limitation required for the massive implantation of these technologies.

Photovoltaic devices represent the most exploded way to harvest solar energy. While there are several generations of photovoltaic devices [106], all of them are based on junctions where electron-holes pairs created by light absorption are separated, leading to charge accumulation that produces electric voltage, i.e., electric energy, as figure 21a illustrates. The main limitation in the development and implantation of photovoltaic devices is their efficiency. Photovoltaic devices result economically viable with cost below $\sim 1 \$ /$ Watt [114], a value scarcely achievable by last generation solar cells [106]. A key problem in improving efficiency of photovoltaic devices is that large thicknesses are required to absorb most of the incoming light, especially for silicon based solar cells, due to the indirect gap nature of silicon. However, these larger thicknesses enhance the electron-hole recombination probability increasing looses and requires larger amount of material (i.e., larger production costs). Thus, the efficiency of solar cells still is an open question: nowadays, the most efficient devices cells reach $25 \%$ of energy conversion efficiency [106].

The incorporation of metallic NPs in solar cells can increase the efficiency of charge separation by several physical processes $[115,116,117]$. As figure 21 illustrates, if we place metallic NPs at the device surface, upon light illumination, the large scattering cross section associated with SP can scatter the normal incident light beams. Hence, the effective path across the active absorption layer in increased. Moreover, with a proper architecture, this scattering may be used to promote total reflection inside the layer trapping the photons until they are finally absorbed (figure 21b). Another method to improve efficiency exploding SP, consists of placing the NPs at the junction interface (figure 21c). The light concentration and local amplification in the vicinity of the NP when SP are excited increases the absorption efficiency. Stuart and Harris [118] 
demonstrated an increase up to a factor 20 in the photocurrent of a silicon photodetector by placing silver NPs on the surface.

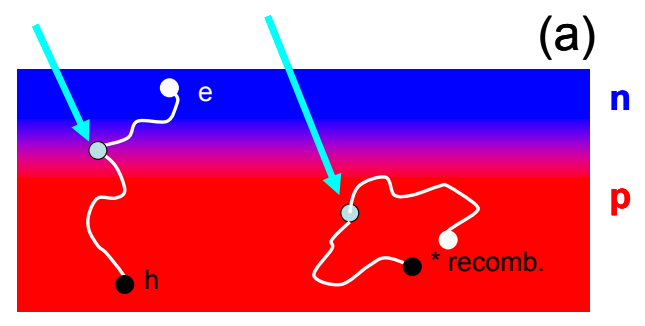

(b)

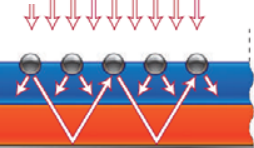

(c)

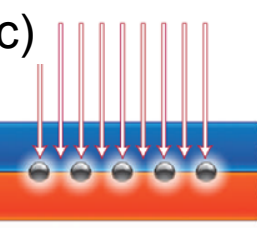

Figure 21 (top) Scheme of photovoltaic cell. Light absorption at the $p$-n junction induces charge separation. If the absorption takes place far from the junction, recombination results more likely, reducing the cell efficiency; (bottom) Illustration of processes improving photovoltaic efficiency by (a) light scattering and (b) local amplification. Reproduced with permission from [115].

Increases of photovoltaic cells efficiency of the order of $10-15 \%$ have been achieved by incorporating Au and Ag NPs on the cell surface $[119,120,121,122,123]$. The efficiency in this kind of devices has a complex dependence on the NPs size, shape and spatial distribution, since interacting effects play a different role on the light scattering and absorption processes [116]. The impact of both mechanisms in the final improvement is not easy to disentangle although most recent results points toward the scattering process as the key one. Actually, some outstanding results have been achieved by using non-resonant silica NPs [124]. The use of SP has been explored not only in silicon based solar cells, but also in third generation organic devices $[125,126,127]$ with efficiency enhancement up to a factor 2 .
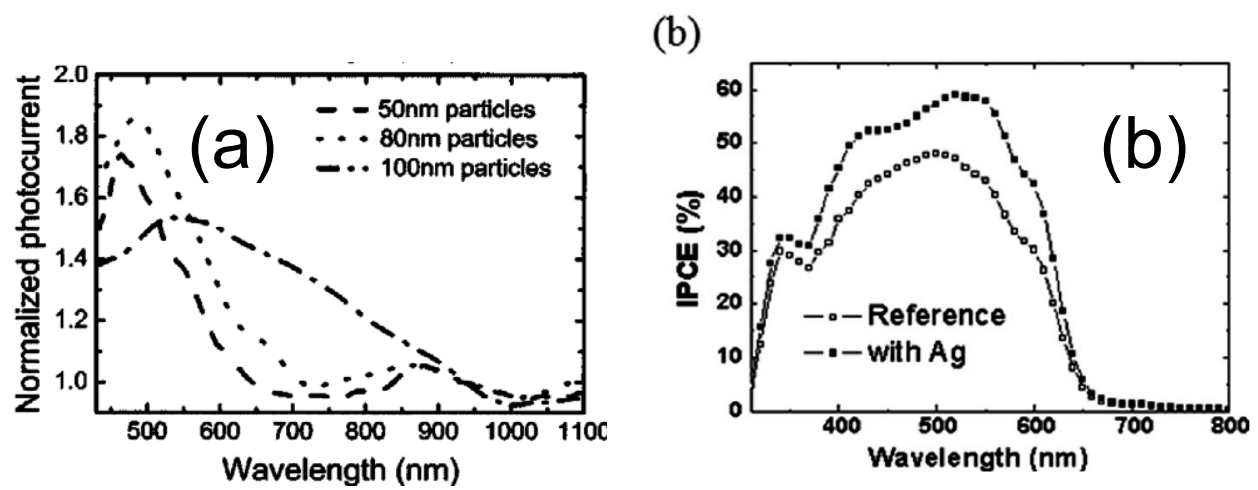

Figure 22. Improvement of efficiency on (a) silicon and (b) organic photovoltaic cells by incorporation of silver NPs. Reproduced with permission from [123] and [126] respectively. 
Photothermal technology is an alternative to photovoltaics especially when the energy is used for heating [128]. In typical photothermal devices, a fluid absorbing light increases its temperature and is submitted to a thermal cycle releasing energy. The efficiency of thermal cycles is limited by the temperature difference between the hot and cold focus. Thus, reducing the mass that absorbs the solar light will increase its temperature and consequently improve the efficiency of the device. The huge extinction cross section of SP provides a method to achieve intense ligh absorption by small masses. Moreover, the tunability of SP resonance band allows the design of selective absorbers that reduces energy looses due to blackbody radiation [129].

Besides the pure physical methods, SP can also be used to catalyse reactions for energy harvesting using chemical paths, for instance the water decomposition for hydrogen generation as described in the next section.

In general, any photoenergetic process addresses the problem to explode the wide solar spectrum. As most of optical transitions in bulk materials exhibit well defined absorption bands, it is difficult to design materials capable to absorb efficiently over a large part of the solar spectrum. SP are not an exception, but, as described above, it is possible to tune the SP band in a wide range, something not straightforward with other absorbing materials as dyes or semiconductors. This tunability rends SP especially advantageous for wide spectrum photo-energetic processes

\subsection{Environment}

The development of technological societies requires a progressive increase of the industrial activity. Many industrial processes result in hazardous products for humans and environment [130]. Reaction of these products to be transformed in inert ones is mandatory in order to ensure a secure and sustainable development of our society. This problem has got particular importance with the recent incorporation to the first world of new regions that increased exponentially their industrial activity in the last years. Catalysis provides a pathway to eliminate these hazardous products quickly, hence reducing their potential danger. Among the different catalytic processes, photocatalysis exhibits particular importance since it is a clean process that can use solar light and mimets other biological processes [131,132,133].

Metallic NPs exhibit catalytic activity [134] that can be improved upon light illumination to excite SP [135]. However, the most exploded use of SP in photocatalysis is the enhancement of photocatalytic efficiency in transition metal oxides by incorporation of metallic NPs. The principle of photocatalysis in these material $[136,137]$ is illustrated in figure 23 . Transition metal oxides (as $\mathrm{TiO}_{2}$ ) exhibit an energy gap in the UV-VIS part of the spectrum. Thus, upon illumination, electrons 
can be promoted to the conduction band. The excited electrons are available to participate in the oxidation of the products that are absorbed on the catalytic surface. Similarly, holes created in the valence band allow the donation of an electron from the absorbed products yielding their reduction. It is straightforward to understand that the efficiency of the catalytic surface (measured as the number of reaction that takes place per unit area and time) will depend critically on the light absorption process that governs the number of active electrons/holes in the material.

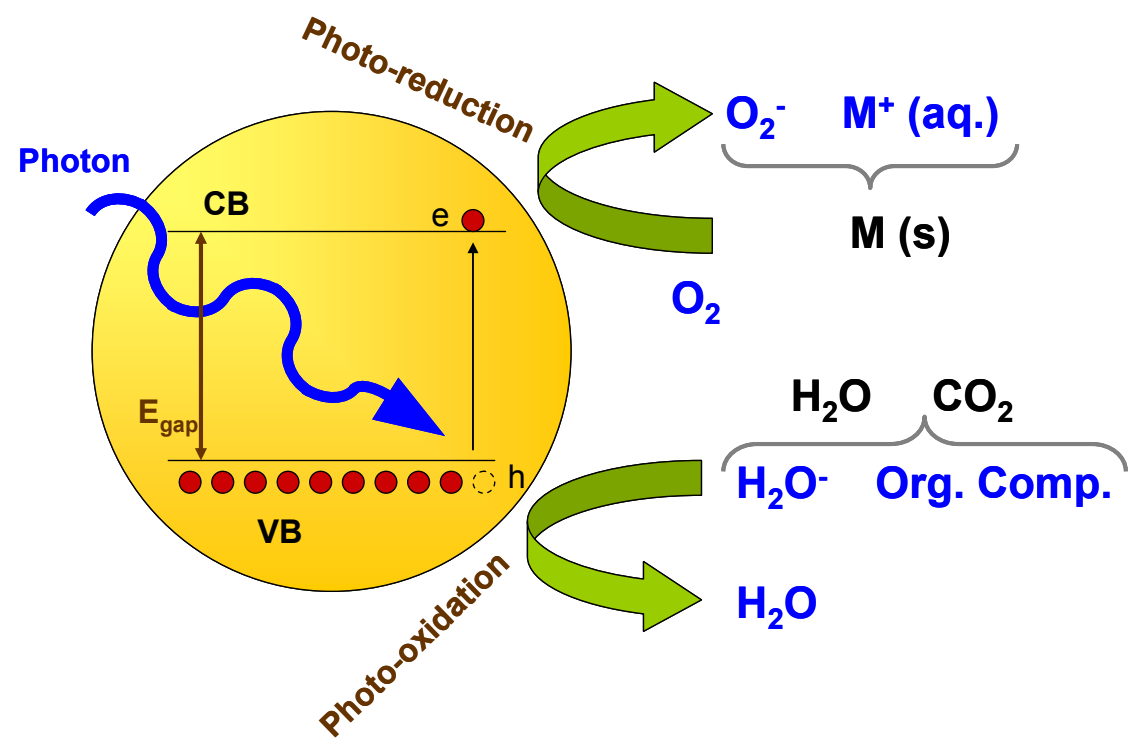

Figure 23. Scheme of the photocatalysis processes in transition metal oxides.

The incorporation of metallic NPs have been used in the past since they act as electron traps retarding electron-hole recombination and therefore increasing the process efficiency $[138,139,140]$. However, the use of NPs exhibiting SP resonance by the photocatalytic surface generates and additional increase of light absorption efficiency by the local electric field amplification and scattering processes as in the photovoltaic devices above described.

Awazu et al [141] demonstrated the increase of photocatalytic efficiency of a $\mathrm{TiO}_{2}$ layer by embedding Ag NPs near the surface as shown in figure 24a. The mechanism of efficiency improvement is basically the same above described for photovoltaic devices (figure 21). The light scattering at the silver NPs increases the effective optical path of photons leading to a higher absorption rate, while the local electric field enhancement also assists the absorption processes. With this arrangement, they found an increase above one order of magnitude in the decomposition rate of methylene blue under near-UV irradiation (figure 24b). Similarly the catalysis of carbon monoxide is substantially improved by the combined use of $\mathrm{Au}$ and $\mathrm{Fe}_{2} \mathrm{O}_{3}$ NPs [142] as shown in figure 24d. While separated use of $\mathrm{Au}$ or $\mathrm{Fe}_{2} \mathrm{O}_{3} \mathrm{NPs}$ leads to almost negligible photocatalytic efficiency, their combined use shows outstanding results activity as the 
excitation of SP in $\mathrm{Au}$ NPs enhances the photocatalysis of $\mathrm{Fe}_{2} \mathrm{O}_{3}$. SP enhancement photocatalysis of $\mathrm{TiO}_{2}$ have been demonstrated for other reaction as methanol dehydrogenation, acetic acid decomposition and propanol oxidation and others [143,144,145,146].

The incorporation of metallic NPs holding SP in photocatalytic materials may have additional advantages. A key problem in some photocatalyst as $\mathrm{ZnO}$ is the photo-instability in aqueous solution due to photocorrosion upon UV illumination [147]. It has been reported that incorporation of $\mathrm{Ag} \mathrm{NPs}$ onto $\mathrm{ZnO}$ films leads to an increase of photocatalytic efficiency and higher stability of $\mathrm{ZnO}$ preventing the photocorrosion [148]. As figure 24c evidences, the presence of Ag NPs avoid the photodegradation upon UV illumination.

SP can be also used to probe catalytic reactions. Larsson et al [149] could monitor $\mathrm{CO}$ and $\mathrm{H}_{2}$ oxidation on $\mathrm{Pt}$, and $\mathrm{NO}_{x}$ conversion to $\mathrm{N}_{2}$ on $\mathrm{Pt} / \mathrm{BaO}$ reaction by following changes in the $\mathrm{SP}$ resonance of Au NPs incorporated in the photocatalytic surface. The high sensitivity of SP to the local environment dielectric properties induces a shift in the SP resonant position when the catalytic process takes place as illustrated in figure $24 \mathrm{e}$. With this method, they were able to detect surfactant coverage with sensitivity below $10^{-3}$ monolayers.

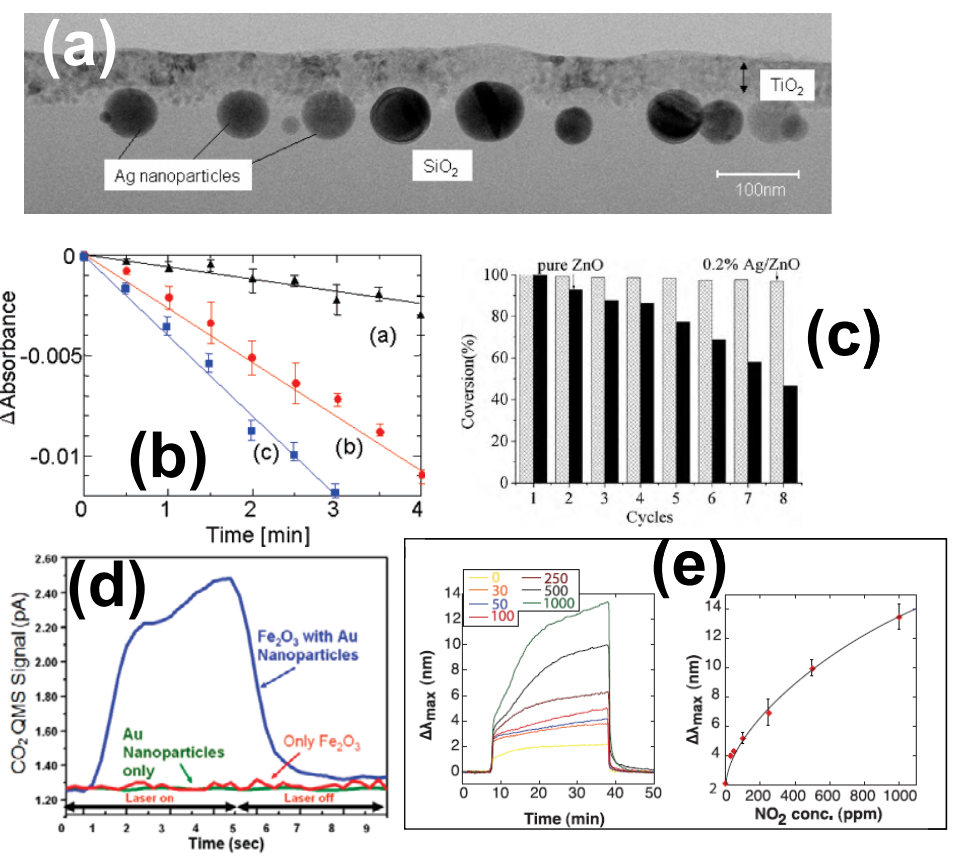

Figure 24 (a) TEM images of $\mathrm{TiO}_{2}$ film on Si with Ag NPs and (b) decomposition rate of methylene blue upon UV irradiation on the $\mathrm{TiO}_{2}$ surface without (black) and with (red\&blue) Ag NPs with different geometry. (c) Durability of $\mathrm{ZnO}$ photocatalysts for the photodegradation of crystal violet under UV irradiation with and without Ag NPs. (d) Photocatalytic $\mathrm{CO}$ decomposition activity using $\mathrm{Fe}_{2} \mathrm{O}_{3}$ and $\mathrm{Au}$ NPs. (e) Shift in the SPR peak of Au NPs during the photo-oxidation of $\mathrm{NO}_{x} \rightarrow \mathrm{NO}_{2}$ on $\mathrm{BaO}$ photocatalyst. Reproduced with permission from [141] (a\&b), [148] (c), [142] (d) and [149] (e). 
A particularly interesting case of photocatalysis is the photo-assisted hydrogen fuel generation. Water decomposition $\left(2 \mathrm{H}_{2} \mathrm{O} \rightarrow 2 \mathrm{H}_{2}+\mathrm{O}_{2}\right)$ provides the possibility to store hydrogen to be used as fuel $[150,151]$. The endothermic decomposition process (that takes place spontaneously at temperatures at about $2500 \mathrm{~K}$ [152]) may be activated by several methods, that increase locally the temperature or catalyse the reaction including chemical, electrical, thermal and optical ones. Thus, solar light can be used to promote water splitting $[152,153]$ using a fully clean and environmental friendly method. As in the abovementioned processes, SP have demonstrated their capabilities to improve the efficiency of the process $[154,155,156$ ]. Liu et al [156] recently found an increase in a factor of 66 on the water splitting photocatalysis by $\mathrm{TiO}_{2}$ when incorporating of Au NPs.

It is worthy mentioning that an indirect improvement of catalytic efficiency using SP may be due to the increase of temperature induced by light absorption. The local enhancement of the temperature associated to SP excitation may help the catalysis process that should be a photothermal assisted catalysis rather than a real photocatalytic one.

\subsection{Information technology}

Optical signals are essential for information technology due to the fast propagation along communication lines and absence of ohmic losses. Light manipulation is commonly achieved by dielectric materials while metals are scarcely used due the short light penetration depth. However, with dielectric materials, it is impossible to focus a beam below $N / 2$ due to the diffraction at the aperture edges [157]. An additional key problem of optical information storage is the difficulty in obtaining re-usable memories since rewritable optical devices stable and mechanically solid are not easy to develop. These restrictions have represented a serious problem for the development of information technology where miniaturization has achieved the nanometric scale. Magnetic memories uses areas below $100 \mathrm{~nm}$ size for bit writing/recording, values that can not be used in traditional optical ones.

Most of the plasmonic applications in this field are achieved with itinerant SP $[2,3]$ but there are also some outstanding examples of localised SP applications for information technologies. The use of nanometals holding SP arises new methods for light manipulating, reading and writing information in sizes significantly smaller than the light wavelength, while the huge absorption and scattering cross sections allows detection of very small signals $[158,159]$. Thus, SP can be used to design subwavelength optical devices as antennas, lenses and resonators for extreme light concentration and manipulation [160,161]. 
The electric field amplification around a metallic NP when SP are excited, make these elements to act as nanoantennas with very low power consumption and large amplification factors $[1,2,3,162]$. Moreover, patterns of NPs with specific geometries provide methods for selective frequency antennas with even larger amplification $[2,3,163,164]$ and to fabricate subwavelength light waveguides with decay length of the order of $500 \mu \mathrm{m}[165,166]$. Photodetectors with improved performance can also be developed exploding SP [167] similarly to the case of photovoltaic cells. The incorporation of metallic NPs in photodetectors increases their efficiency allows to reduce their size $[158,168]$ leading to faster devices with reduced power consumption.

The dependence of SP resonance on factors as size, shape and orientation permits the design of new devices that represent a breakthrough with conventional ones. Recently, Zijlstra et al [169] designated a 5-dimension storage device based on the use of Au NPs and nanorods. Besides the two dimensions of the storage surface, they included the NPs size, aspect ratio and orientation as new ones.

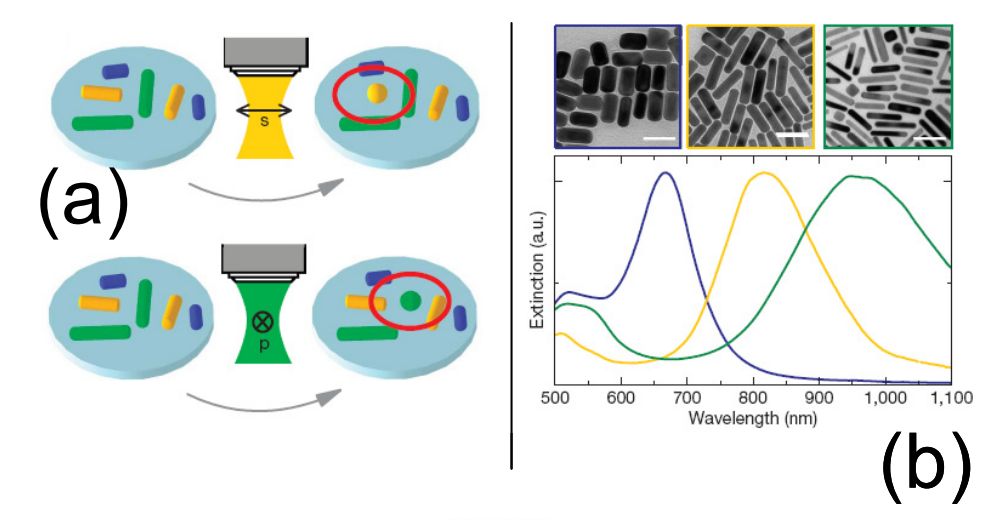

(c)
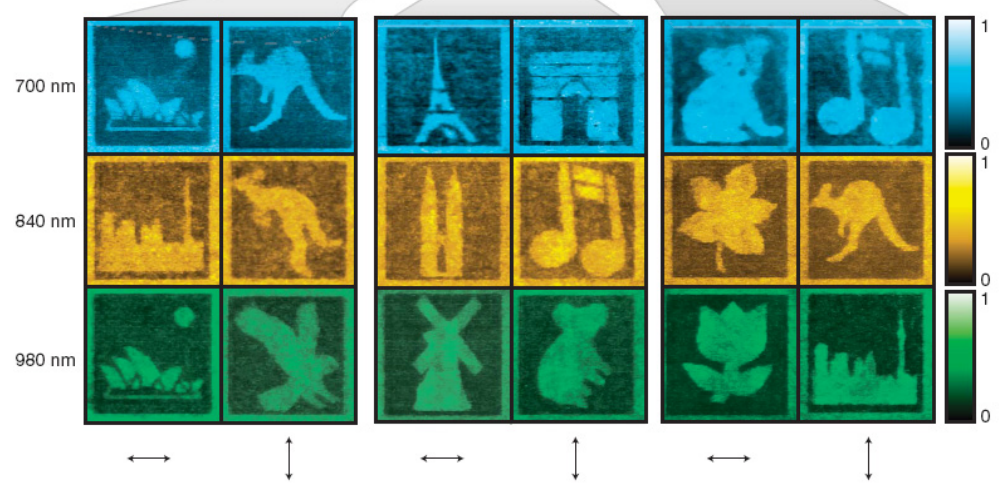

Figure 25 (a) Illustration of the laser induced reshaping of nanorods and (b) TEM images and optical absorption spectra of the nanorods. (c) Images recorded at the same position observed with different light wavelength and polarization. Reproduced with permission from [169]. 
In this device, the information is written using the thermal reshaping of the NPs: When SP are excited with a laser, the local increase of temperature induces a melting that allows to reshape the particles obtaining nanorods with controlled orientation and aspect ratio that depend on the laser wavelength, intensity and polarization $[47,48,49,50]$. Moreover, as the SP band position depends on the NP geometry (figure 25b) it is possible to reshape selectively NPs with certain geometry into other one. Hence, the population of nanorods and their geometrical features can be tuned optically (figure 25a). The information is read using different wavelengths and light polarization to excite selectively some nanorods depending on their morphology. Consequently, it is possible to store several bits of information in the same physical space. With 5 different wavelengths and two different light polarizations they were able to write and read 6 different images in the same area as figure $25 \mathrm{c}$ illustrates with a potential information density of $1 \mathrm{Tby} / \mathrm{cm}^{2}$.

Another example of the potential use of SP for the information technology is the extraordinary light transmission $[170,171,172,173]$. When a uniform light beam reaches an opaque surface with nanometric holes, the light transmission is limited by the diffraction laws. Such a limit can be overcome when dealing with metallic films with holes and SP are excited. The excitation of SP produces a charge accumulation at the holes edge. This lead to a non-uniform wavefront that concentrates the light at the hole region increasing the light transmission.

Excitation of SP can be also used to enhance the sensitivity of Raman spectroscopy by concentrating and locally amplifying the light electric field yielding to much better sensitivity of the technique $[62,174,175,176,177]$. The performance of Scanning Near Field Optical Microscopy (SNOM) is also improved by placing metallic NPs at the tip that concentrates the light $[178,158]$.

\section{Outlook and challenges.}

The excitation of SP in metallic NPs allows exploring regions prohibited for the traditional optics, overcoming the diffraction limit and allowing local light amplification of orders of magnitude. Therefore, potential uses of SP are as ubiquitous as the optics is. These applications include completely new approaches to problems not yet solved and improvement of well established optical processes. Future basic research on SP will certainly prompt new potential applications. However, the nowadays knowledge is solid enough to develop a vast range of applications, some of them already achieved, other in development and some other to be addressed.

The milestone for further development of these applications relays on our ability to process and manufacture nanomaterials. In the case of biomedical applications where economical cost is not a key issue, the challenge is a perfect control of NP size and shape dispersion, as well as 
functionalization. The recent advances in colloidal chemistry pushed the field substantially in the last years to be the most developed among SP applications. For energy and environmental sectors, processing large amounts of NPs with a relatively good control of morphology and size dispersion at competitive costs is the key to promote a widespread use of SP technology. Finally, for information technology, the advances on designing and understanding SP of complex structures and NPs ensembles as well as improvement of lithography methods will drive future advances.

\section{Acknowledgements}

I would like to acknowledge Prof. J. Llopis for lectures and fruitful discussions about surface plasmons during many years. Jose de la Venta is acknowledged for critical reading of the manuscript and valuable comments. This work has been partially supported by Spanish Ministry of Science and innovation through the projects FIS-2008-06249, MAT2010-C21088-C03 and Comunidad de Madrid, project NANOBIOMAGNET (S2009/MAT-1726). 


\section{References}

[1]Kreibig U and Völlmer M, Optical properties of metal clusters ed. Springer-Verlag, Springer Series in Material Science 25 (1995).

[2] Brongersma M L and Kik P G, Surface Plasmon Nanophotonics ed. Springer-Verlag 1988

[3]Maier S A, Plasmonics, ed. Springer-Verlag 2006.

[4] Barnes W L, Dereux A and Ebbesen T W, 2003 Nature 424824

[5]Fritzsche W and Taton T A 2003, Nanotechnology 14 R63.

[6] Hu M, Chen J, Li Z Y, Au L, Hartland G V, Li X, Marquez M and Xia Y, 2006 Chem. Soc. Rev $35,1084$.

[7] S. Eustis and M. A. El-Sayed 2006 Chem Rev. Soc. 35209.

[8] M. A. Garcia, Surface Plamons in biomedicine, in : Recent Developments in BioNanocomposites for Biomedical Applications. A. Tiwari and S. Pilla, editors Novascience Publishers INC, USA, 2010. ISBN 978-1-61761-008-0

[9] Pillai S, Catchpole K R, Trupke T, and Green M A 2007 J. Appl. Phys. 101093105

[10] Atwater H A and Polman A, 2010 Nat. Mat. 9205

[11] Matheu P,Lim S H,Derkacs D, McPheeters C, and Yu E T, 2008 App. Phys. Lett. 93113108

[12] R. Narayanan and M. A. El-Sayed 2005 J. Phys. Chem. B 10912663.

[13] Awazu K, Fujimaki M, Rockstuhl C, Tominaga J, Murakami H, Ohki Y,Yoshida N, Watanabe T, 2008 J. Am. Chem. Soc. 1301676.

[14] Larsson E M, Langhammer C, Zorić I and Kasemo B 2009 Science 3261091

[15] J. Homola (ed.) Surface Plasmon Resonance Based Sensors ed. by Springer Verlag Heidelberg in Springer Series on Chemical Sensors and Biosensors, 2006

[16] Ozbay E, 2006 Science 311189

[17] Fedlheim D L and Foss C A 2001Metal Nanoparticles: Synthesis, Characterization, and Applications CRC Press

[18] Mariotti D and Sankaran R M 2010 J. Phys. D: Appl. Phys. 43323001

[19] Yong K T, Swihart M T, Ding H and Prasad P N, 2009 Plasmonics 479.

[20] Grzelczak M, Pérez-Juste J, Mulvaney P and Liz-Marzán L M, 2008Chem. Soc. Rev. 37 1783

[21] Zhanga Y and Erkey C, 2006 J. of Supercrit. Fluids 38252

[22] Panigrah S, Kundu S, Ghosh S K, Nath S and Pal T 2004, J. of Nanopart. Res. 6411

[23] Goia, D V and Matijevic E 1998 New J. of Chemistry, 221203

[24] Raether H 1988 Surface Plasmons on smooth and rouge surfaces and on gratings. ed. Springer-Verlag 1988.

[25] Sambles J R, Bradbery G W and Yang F 1991 Contemp. Phys. 32173

[26] Mie G, 1908 Ann. Phys. 25377 
[27] Angelini I, Artioli G, Bellintani P, Diella V, Gemmi M, Polla A, Rossi A, 2004 J. of Archaeological Science 311175

[28] D. J. Barber,I. C. Freestone, 1990 Archaeometry 32, 33

[29] I. Freestone, N. Meeks,M. Sax and C. Higgitt , 2007 Gold Bull. 40270

[30] Colomban P, March G, Mazerolles L, Karmous T, Ayed N, Ennabli A and Slim H, $2003 \mathrm{~J}$.

Raman Spectroscopy 34205

[31] Colomban P, 2009 J. of Nano Research 8109

[32]Jose-Yacaman M, Rendon L, Arenas J, Serra Puche M C, 1996 Science 273223

[33] Colomban P and Truong C, 2004 J. Raman Spectrosc. 35195

[34] Brun N, Mazerolles L and Pernot M, 1991 J. Material Science Letters 101418

[35] Nakai I, Numako C, Hosono H and Yamasaki K 1999 J. Amer. Ceram. Soc. 82689.

[36] Wood N, 1999 Chinese Glazes University of Pennsylvania Press, Philadelphia.

[37] Perez-Villar S , Rubio J, Oteo J L, 2008 J. Non-Crystalline Solids, 3541833

[38] Rubio F, Pérez-Villar S, Garrido M A, Rubio J and Oteo J.L J. of Nano Research, 889

[39] Padovani S, Sada C, Mazzoldi P, Brunetti B, Borgia I, Sgamellotti A, Giulivi A, D'Acapito F, and Battaglin G 2003 J. Appl. Phys. 93, 10058

[40] Vilarigues M, Fernandes P, Alves L C, da Silva R C, 2009 Nuclear Instruments and Methods in Physics Research B 2672260

[41] Bobin O, Schvoerer M, Ney C, Rammah M ,Pannequin B,3 Cilia Platamone E, Daoulatli A and Gayraud R P, 2003 Color: Research and Application 28352

[42] Leatherdale C A, Woo W K, Mikulec F V and Bawendi M G 2002 J. Phys. Chem. B 1067619 [43] Jia W, Douglas E P, Guo F and Sun W 2004 Appl. Phys. Lett. 856326

[44] Dresselhaus M S and Dresselhaus G 1962 Phys. Rev. 125499

[45] Mooradian A 1969 Phys. Rev. Lett. 22185.

[46] Dynich R A and Ponyavina A N 2008 J. Appl. Spectr. 75832

[47] Link S, Burda C, Nikoobakht B and El-Sayed M A 200 J. Phys. Chem. B 1046152

[48] Huang W, Qian W and El-Sayed M A 1998 J. Appl. Phys. 98114301

[49] Chang S S, Shih C W, Chen, C. D, Lai W. C and Wang, C R C 1999 Langmuir 15701 (1999)

[50] Link S, Burda C, Nikoobakht, B and El-Sayed, M A 2000 J. Phys. Chem. B 1046152

[51] Lichteneker K 1926 Physik Z. 27115

[52] Maxwell-Garnett J C 1904 Philos. Trans. R. Soc. 203385

[53]Maxwell-Garnett J C 1906 Phil. Trans. R. Soc. 205237

[54] Niklasson G A, Granqvist C G and Hunderi 01981 Applied Optics 2026

[55] Liebsch A and PErsson B N J 1983 J. Phys.C:Solid State Phys. 165375

[56] Bergman D J 1978 Physics Reports 43377 
[57] Garcia M A, Paje S and Llopis J 1999 Chem Phys Lett 315313

[58] Gans R 1912 Ann. Phys, 37881

[59] M. Hu, J. Chen, Z.Y. Li, L. Au, G. V. Hartland, X. Li, M. Marquez and Y. Xia 2006 Chem. Soc. Rev. 351084.

[60] Kreibig U, Schmitz B and Breuer H D 1987 Phys. Rev. B 365027

[61] Hövel H, Fritz D, Holger A ,Kreibig U andVollmer M 1993 Phys. Rev. B 4818178

[62] S. Eustis and M. A. El-Sayed 2006 Chem Rev. Soc. 35209

[63] Kelly K L, Coronado E, Zhao L L and Schatz G C 2003 J. Phys. Chem. B 107668.

[64] Hövel H, Fritz S, Holger A, Kreibig U and Vollmer M 1993 Phys. Rev. B 2118178

[65] Zhang P and Sham T K 2002 Appl. Phys. Lett. 81736

[66] Garcia M A, de la Venta J, Crespo P, LLopis J, Penadés S, Fernández A and Hernando A 2005 Phys. Rev. B 72, 241403

[67] Quinten M and Kreibig U 1986 Surf. Sci. 172557

[68]Westcott S L, Oldenburg S J, Lee T R, Halas N J 1999 Chem Phys. Lett. 300651

[69] Garcia M A 2010 Surface Plasmons in biomedicine in Recent developments in BioNanocomposites for Biomedical Applications, Novascience Publishers, New York

[70] Liao H, Nehl C L and Hafner J H 2006 Nanomedicine 1201

[71] Csáki A, Maubach G, Born D, Reichert J and Fritzsche W 2002 Single Mol. 3275

[72] Fritzsche W and Taton T 2003 Nanotechnology 14 R63

[73] Zu L and Soper S A 2006 Multiplexed Fluorescence detection for DNA sequencing in Reviews in fluorecence 2006, Vol 3, chap 23, Springer

[74] Schena M 2000 Microarray Biochip Technology ed. EATON Pub

[75] Hanfiled J F and Powell R D 2000 J. Histochem. \& Citochem. 48, 471

[76] Bloomfield V A, Crothers D M and Tinoco I 1974 J. Phys. Chem. of Nucleic Acids. chap. 7 , ed. Harper and Row

[77] Yezhelyev M V, Gao X, Al-Haji A, Nie S and O'Regan S M 2006 The Lancet Oncology 7657

[78] Brigger I, Dubernet C and Couvreur P 2002 Adv. Drug Deliv. Rev. 54631.

[79] Li J L, Wang L, Liu X Y, Zhang Z P, Guo H C, Liu W M and Tang S H 2009 Cancer Letters 274319.

[80] El-Sayed I H, Huang X and El-Sayed M A 2005 Nano Letters 5829.

[81] Cai W, Gao T, Hong H and Sun J 2008 Nanotechn., Sci. and Applic. 117

[82] Loo C, Hirsch L, Lee M H, Chang E, West J, Halas N, and Drezek R Opt. Lett. 301012

[83] Sokolov, K, et al. 2003 Cancer Res. 631999

[84] Huang X, El-Sayed I H, Qian W, El-Sayed M A 2006 J. Am. Chem. Soc 1282115

[85] Cai W, Gao T, Hong H and Sun J 2008, Nanotech:Sci and Appl. 117

[86] Jain P K, El-Sayed I H and El-Sayed M A, 2007 NanoToday 218 
[87] Li P C, Wei C W, Liao C K, et al. 2007 IEEE Trans Ultrason Ferroelectr Freq. Control 54 1642

[88] Huang X,El-Sayed I H, Qian W, El-Sayed M A 2007 Nano Lett. 71591

[89] Wabuyele M D, Yan F and Vo-Dinh T 2007 Cellular Imaging and Analysis Using SERS-

Active Nanoparticles Musundi B . Chapter 28 in Nanotechnology in Biology and

MedicineMethods, Devices, and Applications CRC Press

[90] Nitin N, Javier DJ, Richards-Kortum R. Bioconjug Chem, 182090

[91] Eustis S and El-Sayed M A 2006 Chem Rev. Soc. 35209.

[92] Keren S, Zavaleta C, Cheng Z, et al. 2008. Proc. Natl. Acad. Sci. 1055844.

[93]Eghtedari M, Oraevsky A, Copland J a, Kotov N A, Conjusteau A and Motamedi M 2007 Nano Letters 71914.

[94] Van der Zee J 2002 Ann. Oncol. 131173

[95] Huff T B, Tong L, Zhao Y, Hansen M N, Cheng J X and Wei A 2007, Nanomedicine 2125.

[96] Tong L, Wei Q, Wei A and Cheng J X, document avaialble at http://docs.lib.purdue.edu/nanodocs/161

[97] Tong L, Zhao Y, Huff T B, Hansen M N, Wei A and Cheng J X 2007 Adv. Mat. 193136.

[98] Pissuwan D, Valenzuela S M and Cortie M B 2006 TRENDS in Biotechnology 2462.

[99] Kuo T R, Hovhannisyan V A, Chao Y C,Chao S L, Chiang S J, Lin S J, Dong C Y and Chen C C 2010 J. Am. Chem. Soc, 13214163

[100] Yavuz M S, Cheng Y, Chen J, Cobley, C M, Zhang Q, Rycenga M, Xie J, Kim C, Song K H, Schwartz A G, Wang L V and Xia Y. 2009 Nat. Mater. 8935.

[101] Shiotani A, Mori T, Niidome T, Niidome Y, Katayama Y 2007 Langmuir 234012

[102] Podsiadlo P, Sinani V A, Bahng J H, Kam N W S, Lee J, Kotov N A 2008 Langmuir 24568

[103] Ge Z, Kang Y, Taton T A, Braun P V and Cahill D G 2005 Nano Letters 5, 531.

[104] Green M A 2000 Nanotechnology 11401

[105] Marti A and Luque A 2004 Next Generation Photovoltaics, Institute of Physics, London

[106] Green M A Prog. Photovolt: Res. Appl. 200917183

[107] Green M A J Mater Sci: Mater Electron (2007) 18 S15

[108] Litch S 2005 Intern. J. Hydr. Energy 30459

[109] Navarro Yerga R M, Alvarez Galvan M C, del Valle F, Villoria de la Mano J A, and Fierro J L G 2009 Chem. Sus. Chem. 2471

[110] Fendler J H 1985J. Phys. Chem. 892730

[111]Meinel A B and Meinel M P 1976 Environmental Conservation, 315

[112] Khudhair A M and Farid M M 2004 Ener. Conv. and Manag. 45263

[113] Agnihotri O P and Gupta B K 1981 Solar Selective SurfacesWiley-Interscience, New York

[114] Grama S A2007 A survey of thin film solar Photovoltaic Industry \& Technologies PhD Thesis, Massachuset Intitute of Technology 
[115] Atwater H A and Polman A 2010 Nature Materials 9205

[116] Catchpole K R and Polman A 2008 Optics Express 1621793

[117] Pillai S and Green M A 2010 Solar Energy Mat \& Solar Cells 941481

[118] Stuart H R and Hall D G 1998 Appl. Phys. Lett. 733815

[119] Sunadararajan S P, Grady N K, Mirin N and Halas N J 2008 Nano Letters 8624

[120] Pillai S, Catchpole K R, Trupke T and Green M A 2007 J. Appl. Phys. 101093105

[121] Pillai S and Catchpole K R 2006 J. Lumin. 121315

[122] Derkacs D, Lim S H, Matheu P. Mar W and Yu E T 2006 Appl. Phys. Lett. 89093103

[123] Schaadt D M,Feng B and Yu E T 2005 Appl. Phys. Lett. 86063106

[124] Matheu P, Lim S H, Derkacs D, McPheeters C and Yub E T 2008 Appl. Phys. Lett. 93 113108

[125] Morfa A, Rowlen K, Reilly T,Romero M and van de Lagemaat J 2008 Appl. Phys. Lett. 92, 013504

[126] Kim S S, Na S I, Jo J, Kim D Y and Nah Y C 2008 Appl. Phys. Lett. 93, 073307

[127] Reilly III T H,van de Lagemaat J ,Tenent R C,Morfa A and Rowlen K L 2008 Appl. Phys. Lett. 92243304

[128] Seraphin B 01979 Topics in Appl. Phys. 315

[129] Oelhafen P and Schüler A 2005 Solar Energy 79110

[130] Hoffmann M R,Martin S T, Choi W and Bahnemann D W 1995 Chem Rev. 9569

[131] Photcatalyis:Fundamental and applications Serpone N and Pelizzeti E editors 1989 , John Willey\& Son

[132] Herrmann JM 1990 Catalysis Today 53115

[133] Moshfegh A Z 2009 J. Phys. D: Appl. Phys. 42233001

[134] Haruta M and Date M 2001 Applied Catalysis A: General 222427

[135] Zhu H, Chen X, Zheng Z, Ke X, Jaatinen E, Zhao J,Guo C,Xied T and Wang D 2009 Chem Comm. 7524

[136] Linsebigler A L, Lu G and Yates Jr J T 1995 Chem. Rev. 95735

[137] Fujishima A, Hashimoto K and Watanabe $\mathrm{T} 1999 \mathrm{TiO}_{2}$ Photocatalysis Fundaments and Applications, BKC, Inc. Chiyoda-ku, Tokyo

[138] Sreethawong T and Yoshikawa S 2005 Catal. Commun. 6661

[139] Yu Z and Chuang S 2008 Appl. Catal. B, EnViron. 83277.

[140] Chen J J, Wu J C S, Wu P C and Tsai D P 2011 J. Phys. Chem. C 115, 210

[141] Awazu K, Fujimaki M, Rockstuhl C,Tominaga J, Murakami H, Ohki Y, Yoshida N, Watanabe T 2008 J. Am. Chem Soc. 1301676

[142] Hung W H, Aykol M, Valley D, Hou W and Cronin S B 2010 Nano Letters 101314

[143] Kowalska E, Prieto Mahaney O O,Abe R and Ohtani B 2010 Phys. Chem. Chem. Phys. 12 2344 
[144]Reddy V R, Currao A and Calzaferri G 2007 J. Phys: Conf. Series 61960

[145] Valden M, Lai X and Goodman D. W. 1998 Science 2811647

[146] Hammer B and Norskov J. K. 1995 Nature 376238

[147]Jongh P E, Meulenkamp E A, Vanmaekelbergh D and Kelly J J 2000 J. Phys. Chem. B 104 7686

[148] Xie W, Li Y, Sun W, Huang J, Xie H and Zhao X 2010 J. Photochem. \& Photobiol. A: Chem. 216149

[149] Larsson E M, Langhammer C, Zorić I and Kasemo B 2009 Science 3261091

[150] Bard A J and Fox M A 1995 Acc. Chem Res. 28141

[151] Ni M, Leung M K H, Leung D Y C and Sumathy K 2007 Renew. \& Sust. Energy Rev.

11401

[152] Steinfield A 2002 Int.. J. of Hydrogen. Energy 27611

[153] Bard A. J. and Fox M A 1995 Acc. Chem. Res. 28141

[154] Iwasea A, Katoa H and Kudo A 2006 Catal. Lett. 1087

[155]Chen J J, Wu J C S,Wu P C and Tsai D P 2011 J. Phys. Chem. C 115210

[156] Liu Z, Hou W, Pavaskar P, Aykol M and Cronin S B 2011 Nano Lett.

dx.doi.org/10.1021/nl104005n

[157] Born M and Wolf E 1997. Principles of Optics. Cambridge University Press

[158] Schuller J A, Barnard E S, Cai W, Jun Y C, White J S and Brongersma M L 2010 Nature Materials 9193

[159] Sau T K, Rogach A L, Jackel F, Flar T A and Feldman J 2010 Adv. Mat 22105

[160] Barnes W L, Dereux A and Ebbesen T W 2003 Nature 424824

[161] A very complete view is presneted in 2008 New J. Phys. 10

[162]Hewageeganaa P and Stockman M I 2007 Infrared Phys. \& Techn. 50177

[163] Pellegrini G, Mattei G and P Mazzoldi 2009 Nanotech. 20065201

[164] Bakker R M, Drachev V P, Liu Z, Yuan H K, Pedersen R H, Boltasseva A, Chen J,

Irudayaraj J, Kildishev A V and Shalaev V M 2008 New J. Phys. 10125022

[165] Ozbay E 2006 Science 311189

[166] Maier S A, Barclay P E, Johnson T J, Friedman M D and Painter O 2005 Appl. Phys. Lett. 86071103

[167] Howard R. Stuart and Dennis G. Hall 1998 Appl. Phys. Lett. 733815

[168] Barwicz T et al 2007 J. Opt Netw. 663

[169] Zijstra P, Chon J W M and Gu M 2009 Nature 459410

[170] Ebbesen T W, Lezec H J, Ghaemi H F, Thio T and Wolf P A 1998 Nature 391667

[171] Hutter E and Fendler J H 2004 Adv. Mat 161685

[172] Gao H, Henzie J, and Odom T W 2006 Nano Letters 62104 
[173] Chang S H and Gray S K 2005 Opt. Exp. 133150

[174] Willets K A and Van Duyne R P 2007 Ann. Rev. Phys. Chem. 58267

[175] Campion A and Kambhampati P 1998 Chem. Soc. Rev. 27241.

[176] Kneipp K, Kneipp H, Itzkan I, Dasari R R and Feld M S 1999 Chem. Rev. 992957.

[177] Nie S and Emory S R 1997 Science 2751102

[178] Sqalli O, Bernal M P, Hoffmann P and Marquis-Weible F 2000 Appl. Phys. Lett. 762134 